\title{
Computational pharmacogenomic screen identifies drugs that phenocopy dipyridamole and potentiate anti-breast cancer activity of statins
}

\section{Jenna van Leeuwen}

University of Toronto https://orcid.org/0000-0002-4744-2960

\section{Wail Ba alawi}

Princess Margaret Cancer Centre

\section{Emily Branchard}

University Health Network

\section{Joseph Longo}

University of Toronto https://orcid.org/0000-0002-9580-5105

\section{Jennifer Silvester}

Princess Margaret Cancer Centre

\section{David Cescon}

Princess Margaret Cancer Centre, University Health Network https://orcid.org/0000-0002-1080-0998

\section{Benjamin Haibe-Kains}

Princess Margaret Cancer Centre

Linda Penn ( $\sim$ Ipenn@uhnresearch.ca )

University Health Network

\section{Deena Gendoo}

University of Birmingham

\section{Article}

Keywords: drug combinations, cancer therapy, mevalonate pathway, drug similarity, drug perturbations, pharmacogenomics, breast cancer

Posted Date: February 18th, 2021

DOl: https://doi.org/10.21203/rs.3.rs-199688/v1

License: (c) (1) This work is licensed under a Creative Commons Attribution 4.0 International License.

Read Full License 
Version of Record: A version of this preprint was published at Nature Communications on October 24th, 2022. See the published version at https://doi.org/10.1038/s41467-022-33144-9. 
3 Jenna van Leeuwen ${ }^{1,2}$, Wail Ba-Alawi ${ }^{1,2}$, Emily Branchard², Joseph Longo ${ }^{1,2}$, Jennifer Silvester ${ }^{3}$, David W.

4 Cescon $^{2,3,4}$, Ben amin Haibe-Kains ${ }^{1,2,5,6, \S}$, Linda Z. Penn ${ }^{1,2, \S}$, Deena M.A. Gendoo ${ }^{7, \S}$

5 'Department of Medical Biophysics, University of Toronto, 101 College Street, Toronto, ON, Canada, M5G

$61 \mathrm{~L} 7$

7 2Princess Margaret Cancer Centre, University Health Network, 101 College Street, Toronto, ON, Canada, 8 M5G 1 L7

9 3The Campbell Family Institute for Breast Cancer Research (CFIBCR), 620 University Avenue, Toronto,

10 ON, Canada, M5G 2C1

$11{ }^{4}$ Division of Medical Oncology and Hematology, Department of Medicine, University of Toronto, 27 King's

12 College Circle, Toronto, ON, Canada, M5S 1A1

13 DDepartment of Computer Science, University of Toronto, 10 King's College Road, Toronto, ON, Canada, 14 M5S 3G4

$15{ }^{6}$ Ontario Institute of Cancer Research, 661 University Avenue, Suite 510, Toronto, ON, Canada, M5G 0A3

$16{ }^{7}$ Centre for Computational Biology, Institute of Cancer and Genomic Sciences, University of Birmingham,

17 Birmingham, Birmingham B15 2TT, United Kingdom

$18 \S$ Co-corresponding authors

19 Address for correspondence: Regarding computational aspects, Dr. Haibe-Kains

20 <Ben amin.Haibe-Kains@uhnresearch.ca> and Dr. Gendoo <d.gendoo@bham.ac.uk>; regarding statin

21 aspects, Dr. Penn <Linda.Penn@uhnresearch.ca>. 


\section{Abstract}

23 Statins are a family of FDA-approved cholesterol-lowering drugs that inhibit the rate-limiting enzyme of

24 the metabolic mevalonate pathway, which have been shown to have anti-cancer activity. As therapeutic

25 efficacy increases when drugs are used in combination, we sought to identify agents, like dipyridamole,

26 that potentiate statin-induced tumor cell death. As an antiplatelet agent dipyridamole will not be suitable

27 for all cancer patients. Thus, we developed an integrative pharmacogenomics pipeline to identify agents

28 that were similar to dipyridamole at the level of drug structure, in vitro sensitivity and molecular

29 perturbation. To enrich for compounds expected to target the mevalonate pathway, we took a

30 pathway-centric approach towards computational selection, which we called mevalonate drug network

31 fusion (MVA-DNF). We validated two of the top ranked compounds, nelfinavir and honokiol and

32 demonstrated that, like dipyridamole, they synergize with fluvastatin to potentiate tumour cell death by

33 blocking the restorative feedback loop. This is achieved by inhibiting activation of the key transcription

34 factor that induces mevalonate pathway gene transcription, sterol regulatory element-binding protein 2

35 (SREBP2). Mechanistically, the synergistic response of fluvastatin-nelfinavir and fluvastatin-honokiol was

36 associated with similar transcriptomic and proteomic pathways, indicating a similar mechanism of action

37 between nelfinavir and honokiol when combined with fluvastatin. Further analysis identified the canonical

38 epithelial-mesenchymal transition (EMT) gene, E-cadherin as a biomarker of these synergistic responses

39 across a large panel of breast cancer cell lines. Thus, our computational pharmacogenomic approach can

40 identify novel compounds that phenocopy a compound of interest in a pathway-specific manner.

41 Key words: Drug combinations; cancer therapy; mevalonate pathway; drug similarity; drug perturbations;

42 pharmacogenomics; breast cancer 


\section{Significance Statement:}

44 We provide a rapid and cost-effective strategy to expand a class of drugs with a similar phenotype. Our

45 parent compound, dipyridamole, potentiated statin-induced tumour cell death by blocking the

46 statin-triggered restorative feedback response that dampens statins pro-apoptotic activity. To identify

47 compounds with this activity we performed a pharmacogenomic analysis to distinguish agents similar to

48 dipyridamole in terms of structure, cell sensitivity and molecular perturbations. As dipyridamole has many

49 reported activities, we focused our molecular perturbation analysis on the pathway inhibited by statins,

50 the metabolic mevalonate pathway. Our strategy was successful as we validated nelfinavir and honokiol

51 as dipyridamole-like compounds at both the phenotypic and molecular levels. Our developed approach

52 sets the framework for future pathway-centric identification of drug combinations. 


\section{Introduction}

54 Triple-negative breast cancer (TNBC) is an aggressive subtype of breast cancer (BC) that has a poorer

55 prognosis amongst the ma or breast cancer subtypes ${ }^{1}$. This poor prognosis stems from our limited

56 understanding of the underlying biology, the lack of targeted therapeutics, and the associated risk of

57 distant recurrence occurring predominantly in the first two years after diagnosis ${ }^{2}$. Cytotoxic anthracycline

58 and taxane-based chemotherapy regimens remain the primary option for treating TNBC, with other

59 classes of investigational agents in various stages of development. Therefore, novel and effective

60 therapeutics are urgently needed to combat this difficult-to-treat cancer.

61 Altered cellular metabolism is a hallmark of cancer ${ }^{3,4}$ and targeting key metabolic pathways can

62 provide new anti-cancer therapeutic strategies. Aberrant activation of the metabolic mevalonate (MVA)

63 pathway is a hallmark of many cancers, including TNBC, as the end-products include cholesterol and

64 other non-sterol isoprenoids essential for cellular proliferation and survival ${ }^{5-7}$. The statin family of

65 FDA-approved cholesterol-lowering drugs are potent inhibitors of the rate-limiting enzyme of the MVA

66 pathway, 3-hydroxy-3-methylglutaryl-CoA reductase $(\mathrm{HMGCR})^{6}$. Epidemiological evidence shows that

67 statin-use as a cholesterol control agent is associated with reduced cancer incidence ${ }^{8}$ and recurrence ${ }^{9-13}$.

68 Specifically, in BC, a 30-60\% reduction in recurrence is evident amongst statin users, and decreased risk

69 is associated with increased statin duration ${ }^{9,12,14,15}$. We and others have shown preclinically that Estrogen

70 Receptor (ER)-negative BC cell lines, including TNBC, are preferentially sensitive to statin-induced

71 apoptosis ${ }^{16,17}$. Moreover, three preoperative clinical trials investigating lipophilic statins (fluvastatin,

72 atorvastatin) in human BC, showed statin use was associated with reduced tumour cell proliferation and

73 increased apoptosis of high-grade $B C s^{18,19}$. Thus, evidence suggests that statins have potential utility in

74 the treatment of BC, including TNBC.

75 Drug combinations that overcome resistance mechanisms and maximize efficacy have potential

76 advantages as cancer therapy. Blocking the MVA pathway with statins triggers a restorative feedback

77 response that significantly dampens the pro-apoptotic activity of statins ${ }^{20,21}$. Briefly, statin-induced 

depletion of intracellular sterols, triggers the inactive cytoplasmic, precursor form of the transcription factor sterol regulatory element-binding protein 2 (SREBP2) to be processed to the active mature nuclear

80 form, which induces transcription of MVA genes, including HMGCR and the upstream synthase

81 (HMGCS1) $)^{22}$. We have shown that inhibiting SREBP2 using RNAi, or blocking SREBP2 processing using

82 the drug dipyridamole, significantly potentiates the ability of statins to trigger tumor cell death ${ }^{21,23,24}$.

83 Dipyridamole is an FDA-approved antiplatelet agent commonly used for secondary stroke

84 prevention, and since statin-dipyridamole has been co-prescribed for other indications, it may be safely

85 used in the treatment of cancer ${ }^{25}$. However, the exact mechanism of dipyridamole action remains unclear

86 as it has been reported to regulate several biological processes. Moreover, the antiplatelet activity of

87 dipyridamole may be a contraindication for some cancer patients. Thus, to expand this dipyridamole-like

88 class of compounds that can potentiate the pro-apoptotic activity of statins, we employed a

89 pathway-centric approach to develop a computational pharmacogenomics pipeline to distinguish

90 compounds that are predicted to behave similarly to dipyridamole in the regulation of MVA pathway

91 genes. Using this strategy, we identified several potential dipyridamole-like compounds including

92 nelfinavir, an FDA-approved antiretroviral drug and honokiol, a compound isolated from Magnolia spp.,

93 which synergise with statins to drive tumour cell death by blocking the restorative feedback response.

94 Correlation analysis of the statin-compound combination synergy score, with basal mRNA expression

95 across a large panel of $\mathrm{BC}$ cell lines, identified $\mathrm{CDH} 1$ expression as a predictive biomarker of response to

96 these combination therapies. Taken together, we provide a new strategy to identify compounds that

97 behave functionally similar to dipyridamole in an MVA pathway-specific manner and suggest that this

98 approach will have broad utility for compound discovery across a wide variety of drug/pathway

99 interactions.

\section{Results}

\section{Computational pharmacogenomic pipeline identifies dipyridamole-like compounds}

102 We developed a computational pipeline that harnesses high-throughput pharmacogenomics analysis to 103 identify dipyridamole-like compounds that synergise with statins by blocking MVA pathway gene 
104 expression to inhibit cancer cell viability (Figure 1A and 1B). The LINCS-L1000 (L1000) ${ }^{26}$ and NCI-6027

105 datasets were chosen for these studies as they contain cellular drug-response data at the molecular and

106 proliferative levels across a panel of cell lines, respectively. From these datasets we extracted drug

107 structure, drug-induced gene perturbation data (gene expression changes after drug treatment) and

108 drug-cell line sensitivity profiles for the 238 compounds common to both datasets. Treating each level of

109 data as a separate layer, we restricted the drug-gene perturbation layer from the L1000 dataset to only

110 include the six MVA pathway genes present in the L1000 landmark gene set, to enrich for compounds

111 that phenocopy the MVA pathway-specific activity of dipyridamole (Supplemental Figure 1A and Figure

112 1B). With dipyridamole as the reference input, we generated an MVA pathway-specific Drug Network

113 Fusion (MVA-DNF) through the integration of three distinct data layers: drug structure, MVA-specific drug

114 perturbation signatures, and drug-cell line sensitivity profiles. For each of the data layers incorporated into

115 MVA-DNF, a 238x238 drug affinity matrix was generated, indicating drug similarity for a selected drug

116 against all other drugs (further described in methods). Briefly, we first computed similarity between pairs

117 of drug structures using the Tanimoto index, prior to generating the structure affinity matrix. We computed

118 the similarity for every pair of drug sensitivity profiles using the pearson correlation coefficient, prior to

119 generating an affinity matrix for the drug sensitivity layer. To create an affinity matrix for the MVA-specific

120 perturbation layer, we first calculated the pearson correlation coefficient on the drug perturbation

121 signatures that were filtered to include only MVA genes (Figure 1B). By integrating the three affinity

122 matrices using similarity network fusion, and filtering hits using permutation testing, we subsequently

123 identified 23 potential dipyridamole-like compounds that scored as significant (permutation test $p$-value

$124<0.05)$ (Figure 1B, Supplementary Table 1, and further explained in methods). Represented as a

125 network, these hits display strong connectivity to dipyridamole as well as to each other.

We assessed the contribution of the different data layers (drug structure, drug-gene perturbation,

127 and drug-cell line sensitivity) within the MVA-DNF for each of these 23 compounds (Figure 1C). Drug

128 perturbation played a significant role in the selection of novel dipyridamole-like compounds compared to

129 drug sensitivity and drug structure. This reflects upon the specificity of the MVA-DNF towards the MVA

130 pathway, in comparison to a 'global' drug taxonomy that is not MVA pathway-centric. Further assessment

131 of the six MVA-pathway gene expression changes within the drug perturbation signatures highlights 
comparable expression profiles between dipyridamole and the novel dipyridamole-like compounds

\section{(Supplementary Figure 1B).}

To prioritize and further interrogate the identified dipyridamole-like hits we annotated the 23 compounds by reported mechanism of action and potential clinical utility. Two compounds were excluded from further analysis as they were not clinically useful: chromomycin A3, a reported toxin ${ }^{28}$, and cadmium chloride, an established carcinogen ${ }^{29}$. The remaining 21 compounds segregated into ten distinct categories, demonstrating that dipyridamole-like hits identified through our pharmacogenomics pipeline spanned a diverse chemical and biological space (Supplemental Figure 1C, Supplemental Table 1). We sought to validate the five hits that scored as most similar to dipyridamole, which belong to four different categories: RAF/MEK inhibitor (selumetinib); antiretroviral (nelfinavir); anthracycline (doxorubicin, mitoxantrone); and natural product (honokiol). The reliability of our approach is evidenced by previous work by our lab and others that the anthracycline doxorubicin potentiates lovastatin in ovarian cancer cells ${ }^{30}$ and RAF/MEK inhibitors such as PD98059 and more recently selumetinib (AZD6244) have been reported to synergise with statins to potentiate cancer cell death ${ }^{31,32}$. The molecular targeted compound (selumetinib) along with the novel three compounds were advanced for further evaluation (nelfinavir, mitoxantrone and honokiol) (Supplemental Table 1).

\section{Dipyridamole-like compounds induce apoptosis in combination with fluvastatin and block the} sterol-regulated feedback loop of the MVA pathway

To investigate whether the dipyridamole-like compounds could potentiate fluvastatin-induced cell death similar to that of dipyridamole, we first investigated sensitivity to increasing statin exposure in combination with a sub-lethal concentration of the novel dipyridamole-like compounds in two breast cancer cell line models with differential sensitivity to fluvastatin as a single agent (Supplemental Figure 2) ${ }^{16}$. As seen with dipyridamole, we observed similar potentiation of fluvastatin (lower $\mathrm{IC}_{50}$ ) when combined with a sub-lethal concentration of selumetinib, nelfinavir, or honokiol, but not mitoxantrone (Supplemental Figure 3 and Supplemental Figure 4). Therefore, mitoxantrone was no longer pursued as a dipyridamole-like compound. To determine the nature of the anti-proliferative activity of the statin-compound combinations, we evaluated cell death by fixed propidium iodide staining and PARP cleavage with selumetinib, nelfinavir, or honokiol. Our data indicate that all three compounds, at 
160

161

182 To investigate whether the potentiation of fluvastatin by nelfinavir and honokiol has broad applicability and

concentrations that have minimal effects as single agents, phenocopy dipyridamole and potentiate statin-induced cell death (Figure 2).

Mechanistically, statins induce a feedback response mediated by SREBP2 that has been shown to dampen cancer cell sensitivity to statin exposure. Moreover, blocking the SREBP2-mediated feedback response with dipyridamole enhances statin-induced cancer cell death ${ }^{21,23}$. We have shown that dipyridamole blocks the regulatory cleavage and therefore activation of SREBP2, decreasing mRNA expression of SREBP2-target genes of the MVA pathway. As expected, statin treatment induced the expression of SREBP2-target genes, INSIG1, HMGCR and HMGCS1 after $16 \mathrm{hr}$ of treatment, which was blocked by the co-treatment with dipyridamole (Figure 3A, Supplemental Figure 5A). Similarly, nelfinavir and honokiol both phenocopy dipyridamole and block the statin-induced expression of MVA pathway genes (Figure 3A, Supplemental Figure 5A). By contrast, co-treatment with selumetinib did not block the fluvastatin-induced feedback response. Housekeeping gene RPL13A was used as a reference gene for normalizing mRNA between samples and was not altered in the presence of the compounds (Supplemental Figure 5B).

Because SREBP2 is synthesized as an inactive full-length precursor that is activated to the mature nuclear form upon proteolytic cleavage, we used western blot analysis to assess the protein levels of both full-length and mature SREBP2. Nelfinavir and honokiol, but not selumetinib, blocked fluvastatin-induced SREBP2 processing and cleavage similar to that of dipyridamole (Figure 3B-C). This suggests that while selumetinib is a strong potentiator of statin-induced cell death, it does not mimic the action of dipyridamole by blocking the restorative feedback response (Figure 3, Supplemental Figure 5).

\section{Novel statin-compound combinations phenocopy synergistic activity of fluvastatin-dipyridamole} in a breast cancer cell line screen

3 examine the determinants of synergy, we further evaluated these statin-compound combinations across a large panel of 47 breast cancer cell lines. A 5-day cytotoxicity assay (sulforhodamine B assay; SRB) in a $6 \times 10$ dose matrix was used to assess fluvastatin-compound efficacy. As expected, dipyridamole treatment resulted in a dose-dependent decrease in fluvastatin $I_{50}$ value (Supplemental Figure 6A). Similarly, nelfinavir and honokiol treatment also resulted in a dose-dependent decrease in fluvastatin $\mathrm{IC}_{50}$ values 
188 similar to that of dipyridamole (Supplemental Figure 6A). This suggests that our computational

189 pharmacogenomic pipeline predicts compounds that potentiate statin activity similarly to dipyridamole 190 across multiple subtypes of breast cancer cell lines.

191 Next, we evaluated statin-compound synergy using the Bliss Index model derived using

192 SynergyFinder ${ }^{33}$ across the panel of breast cancer cell lines. Like the dose-dependent sensitivity data, we

193 observed that the trend in synergy between fluvastatin-dipyridamole across the 47 breast cancer cell lines

194 was also seen with fluvastatin-nelfinavir and fluvastatin-honokiol (Figure 4A). Since we had previously

195 identified that the basal subtype of breast cancer cell lines were more sensitive to single agent

196 fluvastatin ${ }^{16}$, we evaluated whether basal breast cancer cell lines were similarly more sensitive to the

197 fluvastatin-compound combinations. Using the SCMOD2 subtyping scheme, we evaluated the basal,

198 HER2 and luminal B status of each cell line and determined synergy is independent of BC subtype

199 (Supplemental Figure 6B). This suggests these statin-compound combinations can be applied to

200 multiple breast cancer subtypes as therapeutic options, however a biomarker to distinguish those with

201 high-sensitivity remained unclear.

202 Because the synergy profiles across the three fluvastatin-compound combinations were

203 significantly similar (Fluva-NFV vs Fluva-DP, $\mathrm{B}_{\mathrm{s}}=0.55$, $\mathrm{p}$-value $=7.1 \mathrm{e}-05$; Fluva-HNK vs Fluva-DP, $\mathrm{B}_{\mathrm{s}} \equiv 0.62$,

204 p-value=5.5e-06; Fluva-NFV vs Fluva-HNK, $\mathrm{B}_{\mathrm{s}} \equiv 0.82, \mathrm{p}$-value <22e-16), we next interrogated whether

205 baseline gene and/or protein expression profiles across the cell lines for each of the statin-compound

206 combinations was associated with high-sensitivity and the synergistic response. To further interrogate the

207 similarity between the statin-compound combinations, we correlated the RNA-seq and reverse phase

208 protein array (RPPA) profiles of the 47 breast cancer cell lines ${ }^{34}$ with their synergy scores for each of the

209 statin-compound combinations. These represent the transcriptomic and proteomic state associations with

210 synergy for each combination. We then evaluated the correlation between these associations across the

211 different combinations (Fluva-DP vs Fluva-NFV; Fluva-DP vs Fluva-HNK; Fluva-NFV vs Fluva-HNK)

212 (Figure 4B) and identified a high positive correlation between the combinations on the basis of similar

213 transcriptomic associations (Fluva-NFV vs Fluva-DP, $\mathrm{B}_{\mathrm{s}}=0.73$, $\mathrm{p}$-value <2.2e-16; Fluva-HNK vs Fluva-DP,

$214 \mathrm{~B}_{s} \equiv 0.77$, $\mathrm{p}$-value < 22e-16; Fluva-NFV vs Fluva-HNK, $\mathrm{B}_{s} \equiv 0.87, \mathrm{p}$-value <2.2e-16). This high positive

215 correlation was also seen between these combinations using proteomic (RPPA) and synergy data 
216 (Supplemental Figure 6C) suggesting that similar pathways were associated with the synergistic

217 response to the three statin-compound combinations.

218 To compare the overlap in pathways associated with sensitivity to fluvastatin alone, and synergy

219 between the fluvastatin-compound combinations, a Gene Set Enrichment Analysis (GSEA) using the 220 Hallmark Gene Set Collection was performed ${ }^{35}$. These results showed that enriched pathways were 221 highly similar amongst fluvastatin alone and the fluvastatin-compound combinations with one of the 222 highest scoring enriched pathways being epithelial-mesenchymal transition (EMT) (Figure 4C). To further 223 support this finding and because of the low agreement amongst EMT gene sets, we also evaluated four 224 additional GSEA EMT pathways and observed similar trends between fluvastatin alone and the

225 fluvastatin-compound combinations for each of the EMT gene sets (Supplemental Figure 6D). As we 226 and others have published that mesenchymal-enriched cancer cell lines are more sensitive to statin 227 monotherapy ${ }^{36,37}$, this data suggests that fluvastatin is the primary driver of response to these

228 statin-compound combinations. This is consistent with fluvastatin inhibiting the MVA pathway, triggering

229 the SREBP-mediated feedback response, which in turn is inhibited by the second compound

230 (dipyridamole, nelfinavir or honokiol) in these fluvastatin-compound combinations.

231 We then examined the individual genes within each of the GSEA EMT pathways to identify a 232 biomarker of the synergistic response to the statin-compound combinations. Within the EMT field, gene 233 set signatures have low agreement (Supplemental Figure 7). Previously our lab published a binary 234 classifier of five EMT genes to predict increased sensitivity to statins across 631 cell lines representing 235 multiple cancer types ${ }^{36}$. We evaluated whether this binary five-gene classifier could also predict synergy 236 between the different fluvastatin-compound combinations. The five-gene EMT classifier could predict 237 sensitivity to fluvastatin alone across the panel of breast cancer cell lines (Supplemental Figure 8A), but 238 failed to predict synergy to the fluvastatin-compound combinations (Supplemental Figure 8B). We next 239 interrogated each of the five genes individually. Interestingly, low gene expression and protein levels of 240 E-cadherin $(C D H 1)$, a canonical epithelial state marker, not only predicted sensitivity to fluvastatin but 241 also demonstrated synergy across all three fluvastatin-compound combinations (Figure 5A-B and 242 Supplemental Figure 8C). To validate our findings, we probed for basal E-cadherin protein expression 243 across a panel of nine breast cancer cell lines and showed that synergy to the novel statin-compound 244 combinations is positively associated with low E-cadherin protein expression (Figure 5C-D). Overall, this 
245 data validates that our MVA-DNF pharmacogenomics strategy can successfully distinguish compounds

246 that, like-dipyridamole, can synergize with statins to trigger BC tumour cell death.

\section{Discussion}

248 By blocking the statin-induced restorative feedback response, dipyridamole potentiates statin efficacy to 249 drive tumour cell death ${ }^{21,23}$. However, as the platelet-aggregation activity of dipyridamole may preclude its

250 use in some cancer patients, our goal was to expand this class of agents that potentiate the pro-apoptotic 251 activity of statins. To this end, we developed a novel computational pharmacogenomics pipeline that 252 distinguished compounds that are similar to dipyridamole at the level of structure, MVA pathway gene 253 expression perturbation, and anti-proliferative activity. We identified 23 potential dipyridamole-like 254 compounds and then evaluated several of the top hits for their ability to phenocopy dipyridamole. By this 255 approach, we validated that nelfinavir and honokiol sensitize breast cancer cell lines to statin-induced cell 256 death by blocking the statin-induced restorative feedback loop. Analysis of basal RNA and protein 257 expression identified the canonical EMT gene $C D H 1$ (E-cadherin) as a biomarker of the synergistic 258 response to both statin-nelfinavir and statin-honokiol treatment. Thus, despite the polypharmacology of 259 dipyridamole, the computational pharmacogenomics screen described here successfully identified 260 synergistic statin-compound drug combinations as novel anti-breast cancer therapies.

261 By integrating a computational pharmacogenomics pipeline and cellular validation, we provide a 262 novel, rapid, broadly-adaptable, and inexpensive strategy to distinguish compounds with similar biological 263 activities. We show here that this approach overcomes a ma or problem associated with working with 264 drugs, such as dipyridamole, that possess a complex polypharmacology. Dipyridamole was originally 265 identified for its anti-platelet aggregation activity, but its mechanism of action spans a wide variety of 266 functions. Several activities of dipyridamole have been described including an inhibitor of 267 phosphodiesterases $^{38}$, nucleoside transport ${ }^{39}$ and glucose uptake ${ }^{40}$. By restricting the gene perturbation 268 layer of our pharmacogenomics pipeline to MVA-pathway genes, we successfully circumvented many of 269 these varied functions and focused specifically on identifying dipyridamole-like drugs whose mechanisms 
centre on the mevalonate pathway. This highlights that the computational pharmacogenomics pipeline

271

272

273 which like dipyridamole, inhibit statin-induced SREBP2 cleavage and activation ${ }^{21,23}$. To date, a number of

274 SREBP2 inhibitors have been identified that block SREBP2 processing from its precursor to mature form,

275 including fatostatin, betulin, and xanthohumal (ER-Golgi translocation), PF-429242 (site-1 protease (S1P)

276 cleavage), and nelfinavir and 1,10-phenanthroline (site-2 protease (S2P) cleavage). Additional SREBP2

277 inhibitors include BF175 and tocotrienols that target SREBP2 transcriptional activity and protein stability,

278 respectively. However, other than nelfinavir, these agents are either under development for clinical

279 application or are only used for research purposes. The S2P protease inhibitor nelfinavir (marketed as

280 Viracept) was approved for use in 1997 as an antiviral for the treatment of HIV, and is under evaluation for

281 its utility as an anti-cancer agent ${ }^{41-46}$. This further reinforces that the novel combination of statin-nelfinavir

282 is immediately actionable and should be evaluated without delay. We suggest the fluvastatin-nelfinavir

283 combination is prefered compared to other statins, as distinct cytochrome P450 enzymes are used to

284 process these agents, thereby preventing adverse drug-drug interactions ${ }^{47}$.

285 To the best of our knowledge, this is the first study to report honokiol to synergize with statins in

286 the context of cancer. Honokiol is a natural product commonly used in traditional medicine and has a

287 number of reported mechanisms of action. How honokiol inhibits SREBP2 remains unknown, but this is

288 the first study to interrogate its activity in SREBP2 translocation and gene expression alone and in

289 combination with statins. As honokiol and its derivatives are presently under development, our findings of

290 this new mechanism for honokiol can be incorporated into future analysis of honokiol's structure-activity

291 relationships. Two additional predicted dipyridamole-like compounds tested in this study include

292 selumetinib and mitoxantrone; the former was observed to sensitize breast cancer cells to statin-induced

293 apoptosis, but the latter did not. Selumetinib functions through an SREBP2-independent mechanism,

294 which suggests that not only is the identification of feedback-dependent mechanisms beneficial for cancer

295 treatment, but that additional feedback-independent classes of statin-sensitizers can be identified. This is 
296 particularly relevant, as some multiple myeloma and prostate cancer cell lines have been shown to lack 297 the feedback response $20,21,48$.

298 The data presented here have important clinical implications for statins as anti-cancer agents. 299 Despite encouraging positive results from window-of-opportunity clinical trials in breast cancer using 300 statins as a single-agent, a modest effect was observed with some but not all patients ${ }^{18,19}$. Accordingly, 301 discovery of novel therapeutic combinations is necessary to achieve significant clinical impact. Since 302 nelfinavir is poised for repurposing, and statins have demonstrated anti-cancer activity in early-phase 303 clinical trials ${ }^{18,19,49-54}$, clinical studies to further evaluate the therapeutic benefit of this combination could 304 proceed swiftly. Furthermore, analyses of gene and protein expression data across our large collection of 305 breast cancer cell lines identified a mesenchymal-enriched gene expression profile as highly predictive of 306 sensitivity to all three statin-compound (dipyridamole, nelfinavir or honokiol) combinations. We further 307 showed that $C D H 1$ expression levels served as a biomarker of synergistic response. This reinforces the 308 dipyridamole-like behaviour of nelfinavir and honokiol, identified by our pharmacogenomics pipeline, and 309 creates opportunities for biomarker-guided clinical studies (Figure 5E). We also observed this synergistic 310 response to the combination therapies across multiple subtypes of breast cancer. Previously we had

311 identified the basal-like breast cancer subtype as more sensitive to statins alone; here, we have

312 expanded the scope of statin treatment to encompass the wider breast cancer population which can be 313 distinguished on the basis of $C D H 1$ expression.

314 Collectively, our computational pharmacogenomics pipeline underscores the ability to identify 315 compounds that phenocopy our parent compound of interest (dipyridamole), importantly, in a 316 pathway-specific manner (mevalonate). Our study also provides a strong preclinical rationale to warrant 317 further investigation of the fluvastatin-nelfinavir combination, as well as the utility of $C D H 1$ as a biomarker 318 of response. The availability of these approved, well-tolerated drugs as well as simple methods for 319 assessing $C D H 1$ expression could enable rapid translation of these findings to improve breast cancer 320 outcomes. 


\section{Methods}

322 Our analysis design encompasses both computational identification and refinement of dipyridamole-like 323 compounds, as well as experimental validation of the most promising candidates.

\section{MVA-specific Drug Network Fusion (MVA-DNF).}

325 We developed a computational pharmacogenomic pipeline (MVA-DNF) that facilitates identification of 326 analogues to dipyridamole, by elucidating drug-drug relationships specific to the mevalonate (MVA)

327 pathway. MVA-DNF briefly extends upon some principles of the drug network fusion algorithm we had 328 described previously ${ }^{55}$, by utilizing the similarity network fusion algorithm across three drug taxonomies 329 (drug structures, drug perturbation, and drug sensitivity). Drug structure annotations and drug 330 perturbation signatures are obtained from the LINCS-L1000 datase ${ }^{26}$, and drug sensitivity signatures are 331 obtained from the $\mathrm{NCl}-60$ drug panel ${ }^{27}$. Drug structure annotations were converted into drug similarity 332 matrices by calculating tanimoto similarity measures ${ }^{56}$ and extended connectivity fingerprints ${ }^{57}$ across all 333 compounds, as described previously ${ }^{55}$. We extracted calculated Z-scores from drug-dose response 334 curves for the $\mathrm{NCl}-60$ drug sensitivity profiles, and computed Pearson correlation across these profiles to 335 generate a drug similarity matrix based on sensitivity ${ }^{27}$. We used our PharmacoGx package (version

336 1.6.1) to compute drug perturbation signatures for the $L 1000$ dataset using a linear regression model, as 337 described previously ${ }^{58}$. The regression model ad usts for cell specific differences, batch effects and 338 experiment duration, to generate a signature for the effect of drug concentration on the transcriptional 339 state of a cell. This facilitates identification of gene expression which has been significantly perturbed due

340 to drug treatment. These signatures indicate transcriptional changes that are induced by compounds on 341 cancer cell lines. We further refined the drug perturbation profiles to a set of six MVA-pathway genes

342 (Supplementary Figure 1A) that had been obtained from the literature as well as repositories of 343 pathway-specific gene sets including $\mathrm{MSigDB}^{59}$, HumanCyc ${ }^{60}$ and $\mathrm{KEGG}^{61,62}$. These gene sets include

344 'mevalonate pathway' and 'superpathway of geranylgeranyldiphosphate biosynthesis I (via mevalonate)'

345 from the HumanCyc ${ }^{63}$, and 'Kegg Terpenoid Backbone Biosynthesis' from KEGG61,62. The filtered 
346 drug-induced gene perturbation signatures were subsequently used to generate a drug perturbation

347 similarity matrix that elucidates drug-drug relationships based on common transcriptional changes across

348 the six MVA-pathway genes. We calculated similarity between estimated standardized coefficients of drug

349 perturbation signatures using the Pearson correlation coefficient. Finally, we used the similarity network

350 fusion algorithm ${ }^{64}$ to integrate the affinity matrices for drug structure, drug sensitivity, and MVA-pathway

351 specific drug perturbation profiles, to generate an MVA-pathway specific drug taxonomy (MVA-DNF)

352 spanning 238 compounds.

\section{Identification of analogues to dipyridamole}

354 We interrogated the MVA-DNF taxonomy using a variety of approaches to identify a candidate set of 355 dipyridamole-like compounds. Using MVA-DNF similarity scores, we first generated a ranking of all

356 compounds closest to dipyridamole. We then conducted a permutation test, to assess the statistical

357 relationship of each ranked drug against dipyridamole. Briefly, drug fusion networks were generated 999

358 times across perturbation, sensitivity, and drug structure profiles, each time using a random set of six

359 genes to generate a 'pathway-centric' drug perturbation similarity matrix. Z-scores and p-values were

360 calculated to determine the statistical relevance of a given dipyridamole-like analog in MVA-DNF,

361 compared to the randomly generated networks. From this, we further ranked a list of dipyridamole-like

362 candidate compounds by their statistical significance within MVA-DNF ( $p$-value $<0.05$ ), resulting in

363 identification of 23 candidate dipyridamole analogs.

For each of the dipyridamole analogues we identified, we conducted a similar assessment of

365 significance to identify the relationships of these compounds to dipyridamole and to themselves. A

366 network of dipyridamole-like analogues was rendered using the iGraph R package ${ }^{65}$. Using MVA-DNF

367 similarity scores, we further assessed the contribution of each of the drug layers (structure, sensitivity and

368 perturbation) towards the identification of dipyridamole-like compounds.

We assessed the regulation of gene expression for genes involved in the mevalonate pathway

370 across all of the top-selected dipyridamole analogues, by analyzing the drug-induced transcriptional 
371 profiles (described above) of the selected analogues. To further prioritize the dipyridamole analogues, the

372 candidate compounds were categorized, and compounds that were known toxins or carcinogens were

373 excluded from the analysis (Supplemental Table 1, Supplemental Figure 1C). Top hits from the largest

374 categories were selected for further validation.

\section{Cell culture and compounds}

376 All cell lines were cultured as described previously ${ }^{16,23}$. Briefly, MDA-MB-231 and HCC1937 cells were 377 cultured in Dulbecco's Modified Eagle's Medium (DMEM) and Roswell Park Memorial Institute medium 378 (RPMI), respectively. All media was supplemented with $10 \%$ fetal bovine serum (FBS), 100 units $/ \mathrm{mL}$ 379 penicillin and $100 \mu \mathrm{g} / \mathrm{mL}$ streptomycin. Cell lines were routinely confirmed to be mycoplasma-free using 380 the MycoAlert Mycoplasma Detection Kit (Lonza), and their authenticity was verified by short-tandem 381 repeat (STR) profiling at The Centre for Applied Genomics (Toronto, ON, Canada). Fluvastatin (US

382 Biological F5277-76) was dissolved in ethanol and dipyridamole (Sigma), nelfinavir (Sigma), honokiol 383 (Sigma), mitoxantrone (Sigma) and selumetinib (Selleckchem) were dissolved in DMSO.

\section{Breast cancer cell lines panel}

385 The breast cancer cell line ${ }^{34}$ panel was a generous gift from Dr. Ben amin Neel. RNAseq quantification 386 was done using Kallisto pipeline ${ }^{66}$ using human transcriptome reference hg38.gencodeV23 ${ }^{67}$. RPPA 387 processed data was downloaded from Marcotte et al. $2016^{34}$. SCMOD2 ${ }^{68}$ breast cancer subtypes of these 388 cell lines were obtained using the genefu R package ${ }^{69}$.

\section{Breast cell-line combination viability screen}

390 We used the sulforhodamine B colorimetric (SRB) proliferation assay ${ }^{70}$ in 96 -well plates to determine the 391 dose-response curves. To test the combinations in the panel of BC cell lines (See Breast cancer cell lines 392 panel), the fluvastatin/dipyridamole, fluvastatin/nelfinavir and fluvastatin/honokiol drug combinations were 393 tested in a $6 \times 10$ dose matrix format covering a range of decreasing concentrations of each drug (highest 394 drug dose was $20 \mu \mathrm{M}$ fluvastatin, $20 \mu \mathrm{M}$ dipyridamole, $10 \mu \mathrm{M}$ nelfinavir and $20 \mu \mathrm{M}$ honokiol), along with 
395 all their pairwise combinations, as well as the negative control (EtOH and DMSO). We subtracted the

396 average phosphate-buffer saline (PBS) wells value from all wells and computed the standard deviation

397 and coefficient for each replicate. All individually treated well values were normalized to the control well

398 values. We used Prism (v8.2.0, GraphPad Software) to compute dose-response curves with a bottom

399 constraint equal to 0.

\section{Cell viability assays}

401 3-(4,5-dimethylthiazol-2-yl)-2,5-diphenyltetrazolium bromide (MTT) assays were performed as previously 402 described $^{20}$. Briefly, BC cells were seeded in 750-15,000 cells/well in 96-well plates overnight, then 403 treated in triplicate with $0-400 \mu \mathrm{M}$ fluvastatin for 72 hours. Half-maximal inhibitory concentrations $\left(\mathrm{IC}_{50}\right)$ 404 values were computed from dose-response curves using Prism (v8.2.0, GraphPad Software) with a 405 bottom constraint equal to 0 .

\section{Cell death assays}

407 Cells were seeded at $2.5 \times 10^{5}$ cells/plates and treated the next day as indicated. After 72 hours, cells were 408 fixed in $70 \%$ ethanol for $>24 \mathrm{~h}$, stained with propidium iodide and analyzed by flow cytometry for the 409 sub-diploid (\% pre-G1) DNA population as a measure of cell death as previously described ${ }^{20}$.

\section{Immunoblotting}

411 Cell lysates were prepared by washing cells twice with cold PBS and lysing cells in RIPA buffer (50 mM 412 Tris- $\mathrm{HCl} \mathrm{pH}$ 8.0, $150 \mathrm{mM} \mathrm{NaCl}, 0.5 \%$ sodium deoxycholate, 1\% NP-40, 0.1\% SDS, $1 \mathrm{mM}$ EDTA, protease 413 inhibitors) on ice for $30 \mathrm{~min}$. Lysates were cleared by centrifugation and protein concentrations were 414 determined using the Pierce $660 \mathrm{~nm}$ Protein Assay Kit (Thermo Fisher Scientific). Equal amounts of 415 protein were diluted in Laemmli sample buffer, boiled for 5 min and resolved by SDS-polyacrylamide gel 416 electrophoresis. The resolved proteins were then transferred onto nitrocellulose membranes. Membranes 417 were then blocked for $1 \mathrm{hr}$ in 5\% milk in tris-buffered saline/0.1\% Tween-20 (TBS-T) at room 
418 temperature, then probed with the following primary antibodies in $5 \%$ milk/TBS-T overnight at $4{ }^{\circ} \mathrm{C}$ :

419 SREBP-2 (1:250, BD Biosciences, 557037), p44/42 MAPK (ERK1/2) (1:1000, Cell Signaling Technology, 420 4695), PARP (1:1000, Cell Signaling Technology, 9542L), a-Tubulin (1:3000, Calbiochem, CP06) and 421 E-cadherin (1:1000, Cell Signaling Technology, 3195). Primary antibodies were detected using

422 IRDye-con ugated secondary antibodies and the Odyssey Classic Imaging System (LI-COR Biosciences).

423 Densitometric analysis was performed using ImageJ $1.47 \mathrm{v}$ software.

\section{RNA expression analyses}

425 Total RNA was harvested from sub-confluent cells using TRIzol Reagent (Invitrogen). cDNA was

426 synthesized from 500 ng RNA using SuperScript III (Invitrogen). Quantitative reverse transcription PCR

427 (qRT-PCR) was performed using the ABI Prism 7900HT sequence detection system and TaqMan probes 428 (Applied Biosystems) for HMGCR (Hs00168352), HMGCS1 (Hs00266810), INSIG1 (Hs01650979) and 429 RPL13A (Hs01578913).

\section{Drug combinations synergy analysis}

431 Viability scores were calculated using standard pipelines from PharmacoGx R package ${ }^{58}$ and synergy 432 scores represented by Bliss Index were calculated using SynergyFinder R package ${ }^{33}$. Pearson correlation 433 coefficient was used to measure the associations between the transcriptomic and proteomic states of cell 434 lines and the corresponding synergy scores for each of the combinations. The transcriptomic associations 435 were then used to rank genes for GSEA ${ }^{71}$. The Hallmark gene set collection ${ }^{35}$ was downloaded from $436 \mathrm{MSigDB}^{72}$. The Piano $\mathrm{R}$ package was used to run GSEA analysis ${ }^{73}$. Other EMT related pathways, namely 437 "GO Positive Regulation of Epithelial To Mesenchymal Transition"74, "GO Epithelial To Mesenchymal 438 Transition"74, "SARRIO Epithelial Mesenchymal Transition DN"75, and "SARRIO Epithelial Mesenchymal 439 Transition Up"75, were also downloaded from MSigDB for analysis. 


\section{Data Access}

441 The code and associated tutorial describing how to run the analysis pipeline are publicly available on

442 Github at: https://github.com/DGendoo/MVA_DNF. All software dependencies are available on

443 Bioconductor (BioC) or the Comprehensive Repository R Archive Network (CRAN), and have been listed 444 throughout the methods as applicable.

\section{Authors' contributions}

446 Experimental Design: LZP, DG, BHK, JVL, JS, DWC

447 Acquisition of data: JVL, EB, JL, JS

448 Experimental analysis and interpretation: JVL, LZP, BHK, DG, WB, DWC

449 Conception and design of the pathway-centric MVA-DNF \& permutation pipeline: DG, LZP, BHK, JVL, JL

450 Development of computational methodology: BHK, DG, WB

451 Bioinformatics data analysis and interpretation: DG, WB, BHK

452 Code Development: DG, WB

453 Writing, review, and/or revision of the manuscript: JVL, LZP, DWC, WB, BHK, DG

454 Study supervision: LZP and BHK

455 Approved the manuscript: all authors

\section{Conflict of Interest Statement}

457 DWC serves as a consultant for Agendia, Dynamo Therapeutics, AstraZeneca, Exact Sciences, GSK,

458 Merck, Novartis, Pfizer, Puma, Roche; receives research support (to institution) from GSK, Merck and

459 Pfizer and Roche-Genentech, and holds intellectual property as co-inventor on a patent related to

460 biomarkers for TTK inhibitors. All other authors declare that they have no conflicts of interest. 


\section{Acknowledgements}

462 This study was conducted with the support of the Terry Fox Research Institute-New Frontiers Program

463 Pro ect Grant (1064; LZP, BHK, WB and DWC), the Canada Research Chairs Program (to LZP;

464 950-229872) and Canadian Institutes of Health Research (to LZP; MOP-142263). This work was also

465 supported by the Office of the Assistant Secretary of Defense of Health Affairs, through the Breast Cancer

466 Research Program under Award No. W81XWH-16-1-0068 (to LZP and DWC). Opinions, interpretations, 467 conclusions and recommendations are those of the author and are not necessarily endorsed by the

468 Department of Defense. The authors thank all members of the Penn lab for helpful suggestions and 469 critical feedback. 


\section{Figure Legends}

471 Fig. 1. A schematic of the mevalonate (MVA) pathway and overview of the computational

472 pharmacogenomics workflow. (A) In response to fluvastatin treatment (labelled with 1), MVA pathway

473 end-product levels decrease, triggering an SREBP-mediated feedback response that activates MVA

474 pathway-associated gene expression to restore cholesterol and other non-sterol end-product levels.

475 Dipyridamole (DP) (labelled with 2) blocks the SREBP-mediated feedback response, thereby potentiating

476 fluvastatin-induced cancer cell death. (B) An overview of the computational pharmacogenomics workflow,

477 MVA-DNF, used to identify the top 23 "dipyridamole-like" candidates and visualized as a compound

478 network. MVA-DNF combines drug structure, drug sensitivity, and drug-induced gene perturbation

479 datasets restricted to six MVA pathway-specific genes. Permutation specificity testing was performed to

480 select compounds that have a degree of specificity to the mevalonate pathway and dipyridamole.

481 Statistical significance of compounds similar to dipyridamole was assessed by comparing to 999 networks

482 generated from random selection of six genes within the drug perturbation layer. A network representation

483 of dipyridamole and top 23 statistically-significant ( $p$-value $<0.05)$ "dipyridamole-like" compounds are

484 shown. Each node represents a compound and edges connect compounds based on statistical

485 significance of $p$-value $<0.01$. Darker blue nodes and orange edges represent the compounds connected

486 to dipyridamole, and edge thickness represents the associated p-value between the compounds. (C)

487 Radar plot of the top 23 dipyridamole-like compounds ( $p$-value $<0.05$ ), where the contribution of each

488 individual layer of the MVA-DNF (drug structure, sensitivity, and perturbation) is depicted. Percent

489 contribution of each layer is shown from the center $(0 \%)$ to the outer edges $(100 \%)$.

490 Fig. S1, related to Fig. 1. Additional information regarding drug-induced genotype changes and

491 categorization of top 23 dipyridamole-like compounds. (A) Simplified schematic of the MVA pathway,

492 highlighting the six MVA-pathway genes (in red) in the L1000 database used to restrict the drug-induced

493 gene perturbation layer of the MVA-DNF method. (B) Drug perturbation signatures for dipyridamole and

494 dipyridamole-like compounds, plotted for genes pertaining to the MVA pathway. Similarity between 
compounds based on their overall expression profiles is rendered in the dendrogram. Dipyridamole- and

496 fluvastatin-induced changes shown on the bottom as reference. (C) Categorization of the top 21

497 dipyridamole-like compounds excluding toxins and carcinogenic compounds.

498 Fig. S2. MVA-DNF drug-dose response curves for MDA-MB-231 and HCC1937 breast cancer cell

499 lines to identify a sub-lethal dose of top dipyridamole-like compounds. (A) MDA-MB-231 and (B)

500 HCC1937 cells were treated with a range of doses for 72 hours, and cell viability was determined using

501 an MTT assay. The drug dose-response curves are plotted with a dashed line at $80 \%$ MTT activity

502 indicating a sub-lethal drug dose. Data for an average of three technical replicates are plotted; data reflect

503 the results of a single biological experiment. (C) Table of sub-lethal drug dose and interpolated \% MTT

504 activity for both MDA-MB-231 and HCC1937.

505 Fig. S3. MVA-DNF drug-dose response curves, fluvastatin $I_{50}$ and solvent control values for

506 MDA-MB-231 cells. MDA-MB-231 cells were treated with a range of fluvastatin doses alone or in 507 combination with a sub-lethal dose of dipyridamole $(5 \mu \mathrm{M})$, selumetinib $(0.4 \mu \mathrm{M})$, nelfinavir $(3 \mu \mathrm{M})$, 508 mitoxantrone $(0.01 \mu \mathrm{M})$ or honokiol $(12 \mu \mathrm{M})$ for 72 hours, and cell viability was determined using an MTT 509 assay. The drug dose-response curves, fluvastatin $\mathrm{IC}_{50}$ values and control values are plotted. Error bars 510 represent the mean $+/-\mathrm{SD}, \mathrm{n}=3-5,{ }^{*} \mathrm{p}<0.05,{ }^{* *} \mathrm{p}<0.01$ (Student $t$ test, unpaired, two-tailed).

511 Fig. S4. MVA-DNF drug-dose response curves, fluvastatin $I_{50}$ and solvent control values for 512 HCC1937 cells. HCC1937 cells were treated with a range of fluvastatin doses alone or in combination 513 with a sub-lethal dose of dipyridamole $(5 \mu \mathrm{M})$, selumetinib $(1 \mu \mathrm{M})$, nelfinavir $(3 \mu \mathrm{M})$, mitoxantrone $(0.001$ $514 \mu \mathrm{M})$ or honokiol $(10 \mu \mathrm{M})$ for 72 hours, and cell viability was determined using an MTT assay. The drug 515 dose-response curves, fluvastatin $\mathrm{IC}_{50}$ values and control values are plotted. Error bars represent the 516 mean +/- SD, $n=3-6,{ }^{*} p<0.05,{ }^{* *} p<0.01,{ }^{* * *} p<0.001$ (Student $t$ test, unpaired, two-tailed). 
517 Fig. 2. Dipyridamole-like compounds potentiate fluvastatin-induced cell death. (A) MDA-MB-231

518 and HCC1937 cells were treated with solvent controls or fluvastatin +/- dipyridamole (DP), nelfinavir

519 (NFV), honokiol (HNK) or selumetinib (Selu) for 72 hours, fixed in ethanol and assayed for DNA

520 fragmentation (\% pre-G1 population) as a marker of cell death by propidium iodide staining. Error bars

521 represent the mean $+/-\mathrm{SD}, \mathrm{n}=3-4,{ }^{*} \mathrm{p}<0.05,{ }^{* *} \mathrm{p}<0.01,{ }^{* * *} \mathrm{p}<0.0001$ (one-way ANOVA with

522 Bonferroni's multiple comparisons test, where each treatment was compared to the solvent control). (B)

523 Cells were treated as in (A), protein isolated and immunoblotting was performed to assay for PARP

524 cleavage. (F) represents full-length PARP and (C) represents cleaved PARP. (C) PARP cleavage

525 (cleaved/full-length) shown in (B) was quantified by densitometry and normalized to Tubulin expression.

526 Error bars represent the mean $+/-\mathrm{SD}, \mathrm{n}=3-5,{ }^{*} \mathrm{p}<0.05,{ }^{* *} \mathrm{p}<0.005,{ }^{* \star *} \mathrm{p}<0.001,{ }^{\star \star * *} \mathrm{p}<0.0001$ (one-way

527 ANOVA with Bonferroni's multiple comparisons test, where each group was compared to the solvent 528 control within each experiment).

529 Fig. 3. Nelfinavir and Honokiol block fluvastatin-induced SREBP activation. (A) MDA-MB-231 and

530 HCC1937 cells were exposed to solvent controls, fluvastatin (Flu) +/- dipyridamole (DP), nelfinavir (NFV),

531 honokiol (HNK) or selumetinib (Selu) for 16 hours, and RNA was isolated to assay INSIG1 expression by

532 qRT-PCR. mRNA expression data are normalized to RPL13A expression. Error bars represent the mean $533+/-\mathrm{SD}, \mathrm{n}=3-4,{ }^{*} \mathrm{p}<0.05,{ }^{* *} \mathrm{p}<0.005,{ }^{* * *} \mathrm{p}<0.001,{ }^{* * * *} \mathrm{p}<0.0001$ (one-way ANOVA with Bonferroni's

534 multiple comparisons test, where each group was compared to the solvent control group within each

535 experiment). (B) MDA-MB-231 and HCC1937 cells were treated with fluvastatin +/- dipyridamole,

536 nelfinavir, honokiol or selumetinib for 12 hours, and protein was harvested to assay for SREBP2

537 expression and cleavage (activation) by immunoblotting. (P) represents precursor, full-length SREBP2

538 and (M) represents mature, cleaved SREBP2. (C) SREBP2 cleavage (cleaved/full-length) was quantified

539 by densitometry and normalized to total ERK expression. Error bars represent the mean $+/-\mathrm{SD}, \mathrm{n}=3-8$,

$540{ }^{*} \mathrm{p}<0.05,{ }^{* *} \mathrm{p}<0.005,{ }^{* * *} \mathrm{p}<0.001,{ }^{* * *} \mathrm{p}<0.0001$ (one-way ANOVA with Bonferroni's multiple comparisons

541 test, where each group was compared to the solvent controls group within its experiment). 
542 Fig. S5, related to Fig 3. Nelfinavir and Honokiol block fluvastatin-induced SREBP activation of

543 SREBP2 feedback genes. (A) MDA-MB-231 cells were treated with fluvastatin +/- dipyridamole,

544 nelfinavir, honokiol or selumetinib for 16 hours, and RNA was isolated to assay for HMGCR and HMGCS1

545 expression by qRT-PCR. mRNA expression data are normalized to RPL13A expression. (B) RPL13A Ct

546 mean values plotted as a control. Error bars represent the mean $+/-\mathrm{SD}, \mathrm{n}=3-4,{ }^{*} \mathrm{p}<0.05,{ }^{* *} \mathrm{p}<0.005$,

$547^{* * *} \mathrm{p}<0.001,{ }^{* * * *} \mathrm{p}<0.0001$ (one-way ANOVA with Bonferroni's multiple comparisons test, where each

548 group was compared to the solvent controls group).

549 Fig. S6, related to Fig 4. High-throughput compound combination screen. (A) Heatmap of

$550 \log _{10}\left(\right.$ Fluvastatin $\left.\mathrm{IC}_{50}\right)$ values for a high-throughput compound synergy screen against $47 \mathrm{BC}$ cell lines 551 visualizing the $15^{\text {th }}$ to $85^{\text {th }}$ percentile. BC cell lines were treated with a dose matrix of fluvastatin $(0-20 \mu \mathrm{M})$ $552+/$ - dipyridamole (DP) $(0-20 \mu \mathrm{M})$, nelfinavir (NFV) $(0-10 \mu \mathrm{M})$ or honokiol (HNK) $(0-20 \mu \mathrm{M})$. After 5 days of 553 drug treatment, cell viability was assessed by SRB assay. SCMOD2 cell line subtyping was assigned to 554 the BC cell line panel. Data presented are the average of 2 biological replicates (fluvastatin +/555 dipyridamole (DP)) or the mean of 3-6 biological replicates (fluvastatin +/- nelfinavir (NFV) and fluvastatin 556 +/- honokiol (HNK)). (B) Comparison of synergy scores stratified by BC subtypes across the combinations 557 using wilcoxon paired rank test. Red dash line at synergy threshold where $>0$ indicates lower synergy and $558<0$ indicates higher synergy. (C) Associations of proteomic states ${ }^{34}$ with synergy scores across the 559 fluvastatin-compound combinations. Similarity of proteomic states associations were compared across 560 the combinations (Fluva-DP vs Fluva-NFV; Fluva-DP vs Fluva-HNK; Fluva-NFV vs Fluva-HNK) using 561 Pearson correlation coefficient. Top five basally-expressed proteins associated with synergy in either 562 direction are annotated in red. (D) Gene set enrichment analysis using five EMT gene set collections and 563 genes ranked by basal mRNA correlated to the fluvastatin $\mathrm{IC}_{50}$ (Fluva) value or synergy score (Fluva-DP, 564 Fluva-NFV and Fluva-HNK). Dot size indicates the difference in enrichment scores (ES) of the pathways. 565 Background shading indicates the FDR. X indicates pathway and drug combinations that were not 566 significantly enriched (FDR $>0.05)$. 
567 Fig. 4. Compound combination synergy analysis. (A) Heatmap of synergy scores (Bliss Index model)

568 for fluvastatin (Fluva) + dipyridamole (DP), nelfinavir (NFV) or honokiol (HNK) in a panel of 47 breast

569 cancer cells lines. Ordered by synergy score of Fluva-DP, from greatest $(<0)$ to least synergy $(>0)$. Breast

570 cancer subtype of each cell line is shown and is based on the SCMOD2 subtyping scheme. (B) Basal

571 mRNA expression ${ }^{34}$ associations with synergy scores for each drug combination (e.g. Fluva-NFV vs.

572 Fluva-DP, Fluva-HNK vs. Fluva-DP, and Fluva-NFV vs. Fluva-HNK). Associations were calculated using

573 Pearson correlation coefficient. Top five basally-expressed genes associated with synergy in either

574 direction are annotated in red. (C) Gene set enrichment analysis (GSEA) using the Hallmark gene set

575 collection, where genes were ranked according to their correlation to the fluvastatin $\mathrm{IC}_{50}$ (Fluva) value or

576 to the synergy score (Fluva-DP, Fluva-NFV and Fluva-HNK). Dot plot was restricted to pathways enriched

577 in two out of the four groups. Dot size indicates the difference in enrichment scores (ES) of the pathways.

578 Background shading indicates the FDR. X indicates pathway and drug combinations that were not

579 significantly enriched (FDR > 0.05).

580 Fig. S7, related to Fig 4. Overlapping genes within the EMT gene sets. (A) Upset plot to visualize the 581 agreement between Yu et al. (2018) ${ }^{36}$ five-gene classifier and five additional EMT gene sets.

582 Fig. S8, related to Fig 5. EMT gene expression as a biomarker of sensitivity to fluvastatin and

583 synergistic response to fluvastatin-compound combinations. (A) Five-gene fluvastatin sensitivity

584 gene classifier $^{36}$ predicts sensitivity to fluvastatin alone, but (B) does not predict synergy to Fluva-DP,

585 Fluva-NFV or Fluva-HNK. (C) Basal Vimentin (VIM), N-Cadherin (CDH2), ZEB1 and fibronectin (FN1)

586 mRNA expression do not predict synergy to the drug combinations.

587 Fig. 5. Basal E-cadherin predicts synergistic response to fluvastatin-compound combinations. (A)

588 Basal E-cadherin mRNA expression between cell lines predicted to be synergistic or not to each drug

589 combination. Synergy was defined by Bliss Index and significance was measured by wilcoxon rank sum

590 test. (B) Basal E-cadherin mRNA expression between cell lines predicted to be respondent or not to 
591 fluvastatin. Sensitivity was defined by $\mathrm{IC}_{50}$ and significance was measured by wilcoxon rank sum test. (C)

592 Protein lysates were isolated from a panel of breast cancer cell lines to assay for basal E-cadherin

593 expression by immunoblotting. (D) Densitometry values of normalized E-cadherin expression plotted as a

594 heatmap. E-cadherin expression was quantified by densitometry and normalized individually to Tubulin

595 expression. (E) Schematic diagram detailing the potential for fluvastatin (labelled with 1) and nelfinavir

596 (labelled with 2) to block the SREBP2-mediated feedback response and synergize to potentiate

597 fluvastatin-induced cell death.

598 Table Legends

599 Supplementary Table 1 - Ranked MVA-DNF compounds. Z-score and p-values are indicated.

600 Compounds are ordered by $p$-value. 


\section{References}

602 1. Canadian Cancer Society. Canadian Cancer Statistics publication. www.cancer.ca

$603 \mathrm{http}: / /$ www.cancer.ca/en/cancer-information/cancer-101/canadian-cancer-statistics-publication/?regio

$604 \mathrm{n}=$ on (2019).

605 2. Lebert, J. M., Lester, R., Powell, E., Seal, M. \& McCarthy, J. Advances in the systemic treatment of

606 triple-negative breast cancer. Curr. Oncol. 25, S142-S150 (2018).

607 3. DeBerardinis, R. J. \& Chandel, N. S. Fundamentals of cancer metabolism. Science Advances vol. 2 $608 \quad$ e1600200 (2016).

609 4. Warburg, O. On the origin of cancer cells. Science 123, 309-314 (1956).

610 5. Ehmsen, S. et al. Increased Cholesterol Biosynthesis Is a Key Characteristic of Breast Cancer Stem 611 Cells Influencing Patient Outcome. Cell Rep. 27, 3927-3938.e6 (2019).

612 6. Mullen, P. J., Yu, R., Longo, J., Archer, M. C. \& Penn, L. Z. The interplay between cell signalling and 613 the mevalonate pathway in cancer. Nat. Rev. Cancer 16, 718-731 (2016).

6147. Clendening, J. W. et al. Dysregulation of the mevalonate pathway promotes transformation. Proc. $615 \quad$ Natl. Acad. Sci. U. S. A. 107, 15051-15056 (2010).

616 8. Boudreau, D. M. et al. A validation study of patient interview data and pharmacy records for 617 antihypertensive, statin, and antidepressant medication use among older women. Am. J. Epidemiol. $618 \quad 159,308-317$ (2004).

619 9. Ahern, T. P. et al. Statin prescriptions and breast cancer recurrence risk: a Danish nationwide 620 prospective cohort study. J. Natl. Cancer Inst. 103, 1461-1468 (2011).

621 10. Cronin-Fenton, D. et al. Breast cancer recurrence, bone metastases, and visceral metastases in 622 women with stage II and III breast cancer in Denmark. Breast Cancer Res. Treat. 167, 517-528 623 (2018).

624 11. Nielsen, S. F., Nordestgaard, B. G. \& Bo esen, S. E. Statin Use and Reduced Cancer-Related 625 Mortality. New England Journal of Medicine vol. 367 1792-1802 (2012).

626 12. Kwan, M. L., Habel, L. A., Flick, E. D., Quesenberry, C. P. \& Caan, B. Post-diagnosis statin use and 
627 breast cancer recurrence in a prospective cohort study of early stage breast cancer survivors. Breast

628 Cancer Res. Treat. 109, 573-579 (2008).

629 13. Brewer, T. M. et al. Statin use in primary inflammatory breast cancer: a cohort study. Br. J. Cancer $630 \quad 109,318-324(2013)$.

631 14. Chae, Y. K. et al. Reduced risk of breast cancer recurrence in patients using ACE inhibitors, ARBs, 632 and/or statins. Cancer Invest. 29, 585-593 (2011).

633 15. Boudreau, D. M. et al. Comparative safety of cardiovascular medication use and breast cancer 634 outcomes among women with early stage breast cancer. Breast Cancer Res. Treat. 144, 405-416 635 (2014).

636 16. Goard, C. A. et al. Identifying molecular features that distinguish fluvastatin-sensitive breast tumor 637 cells. Breast Cancer Res. Treat. 143, 301-312 (2014).

638 17. Kimbung, S., Lettiero, B., Feldt, M., Bosch, A. \& Borgquist, S. High expression of cholesterol 639 biosynthesis genes is associated with resistance to statin treatment and inferior survival in breast 640 cancer. Oncotarget 7, 59640-59651 (2016).

641 18. Garwood, E. R. et al. Fluvastatin reduces proliferation and increases apoptosis in women with high 642 grade breast cancer. Breast Cancer Res. Treat. 119, 137-144 (2010).

643 19. B arnadottir, O. et al. Targeting HMG-CoA reductase with statins in a window-of-opportunity breast 644 cancer trial. Breast Cancer Res. Treat. 138, 499-508 (2013).

645 20. Clendening, J. W. et al. Exploiting the mevalonate pathway to distinguish statin-sensitive multiple 646 myeloma. Blood 115, 4787-4797 (2010).

647 21. Longo, J. et al. An actionable sterol-regulated feedback loop modulates statin sensitivity in prostate 648 cancer. Mol Metab 25, 119-130 (2019).

649 22. Brown, M. S. \& Goldstein, J. L. The SREBP Pathway: Regulation of Cholesterol Metabolism by 650 Proteolysis of a Membrane-Bound Transcription Factor. Cell vol. 89 331-340 (1997).

651 23. Pandyra, A. et al. Immediate utility of two approved agents to target both the metabolic mevalonate 652 pathway and its restorative feedback loop. Cancer Res. 74, 4772-4782 (2014).

653 24. Pandyra, A. A. et al. Genome-wide RNAi analysis reveals that simultaneous inhibition of specific 
654 mevalonate pathway genes potentiates tumor cell death. Oncotarget 6, 26909-26921 (2015).

655 25. Ye, Y. et al. Enhanced cardioprotection against ischemia-reperfusion in ury with a dipyridamole and

656 low-dose atorvastatin combination. Am. J. Physiol. Heart Circ. Physiol. 293, H813-8 (2007).

657 26. Subramanian, A. et al. A Next Generation Connectivity Map: L1000 Platform and the First 1,000,000

$658 \quad$ Profiles. Cell 171, 1437-1452.e17 (2017).

659 27. Shoemaker, R. H. The NCl60 human tumour cell line anticancer drug screen. Nat. Rev. Cancer $\mathbf{6}$, 660 813-823 (2006).

661 28. Samal, B. et al. Chromomycin A3 for advanced breast cancer: a Southwest Oncology Group study. $662 \quad$ Cancer Treat. Rep. 62, 19-22 (1978).

663 29. Godt, J. et al. The toxicity of cadmium and resulting hazards for human health. J. Occup. Med. 664 Toxicol. 1, 22 (2006).

665 30. Martirosyan, A., Clendening, J. W., Goard, C. A. \& Penn, L. Z. Lovastatin induces apoptosis of 666 ovarian cancer cells and synergizes with doxorubicin: potential therapeutic relevance. BMC Cancer $667 \quad 10,103(2010)$.

668 31. Wu, J., Wong, W. W.-L., Khosravi, F., Minden, M. D. \& Penn, L. Z. Blocking the Raf/MEK/ERK 669 pathway sensitizes acute myelogenous leukemia cells to lovastatin-induced apoptosis. Cancer Res. $670 \quad 64,6461-6468$ (2004).

671 32. McGregor, G. H. et al. Targeting the Metabolic Response to Statin-Mediated Oxidative Stress

672 Produces a Synergistic Antitumor Response. Cancer research vol. 80 175-188 (2020).

673 33. lanevski, A., He, L., Aittokallio, T. \& Tang, J. SynergyFinder: a web application for analyzing drug 674 combination dose-response matrix data. Bioinformatics 33, 2413-2415 (2017).

675 34. Marcotte, R. et al. Functional Genomic Landscape of Human Breast Cancer Drivers, Vulnerabilities, 676 and Resistance. Cell 164, 293-309 (2016).

677 35. Liberzon, A. et al. The Molecular Signatures Database (MSigDB) hallmark gene set collection. Cell $678 \quad$ Syst 1, 417-425 (2015).

679 36. Yu, R. et al. Statin-Induced Cancer Cell Death Can Be Mechanistically Uncoupled from Prenylation of 680 RAS Family Proteins. Cancer Res. 78, 1347-1357 (2018). 
681 37. Viswanathan, V. S. et al. Dependency of a therapy-resistant state of cancer cells on a lipid

682 peroxidase pathway. Nature 547, 453-457 (2017).

683 38. Bender, A. T. \& Beavo, J. A. Cyclic Nucleotide Phosphodiesterases: Molecular Regulation to Clinical

$684 \quad$ Use. Pharmacological Reviews vol. 58 488-520 (2006).

685 39. King, A. E., Ackley, M. A., Cass, C. E., Young, J. D. \& Baldwin, S. A. Nucleoside transporters: from 686 scavengers to novel therapeutic targets. Trends Pharmacol. Sci. 27, 416-425 (2006).

687 40. Steinfelder, H. J. \& Joost, H. G. Inhibition of insulin-stimulated glucose transport in rat adipocytes by 688 nucleoside transport inhibitors. FEBS Letters vol. 227 215-219 (1988).

689 41. Chow, W. A., Jiang, C. \& Guan, M. Anti-HIV drugs for cancer therapeutics: back to the future? Lancet 690 Oncol. 10, 61-71 (2009).

691 42. Guan, M., Su, L., Yuan, Y.-C., Li, H. \& Chow, W. A. Nelfinavir and nelfinavir analogs block site-2 692 protease cleavage to inhibit castration-resistant prostate cancer. Sci. Rep. 5, 9698 (2015).

693 43. Soprano, M. et al. Oxidative Stress Mediates the Antiproliferative Effects of Nelfinavir in Breast $694 \quad$ Cancer Cells. PLoS One 11, e0155970 (2016).

695 44. Hitz, F. et al. Nelfinavir and lenalidomide/dexamethasone in patients with lenalidomide-refractory 696 multiple myeloma. A phase I/II Trial (SAKK 39/10). Blood Cancer J. 9, 70 (2019).

697 45. Rengan, R. et al. Clinical Outcomes of the HIV Protease Inhibitor Nelfinavir With Concurrent 698 Chemoradiotherapy for Unresectable Stage IIIA/IIIB Non--Small Cell Lung Cancer: A Phase 1/2 Trial. 699 JAMA oncology 5, 1464-1472 (2019).

700 46. Blumenthal, G. M. et al. A phase I trial of the HIV protease inhibitor nelfinavir in adults with solid 701 tumors. Oncotarget 5, 8161-8172 (2014).

702 47. Hsyu, P.-H., Schultz-Smith, M. D., Lillibridge, J. H., Lewis, R. H. \& Kerr, B. M. Pharmacokinetic 703 Interactions between Nelfinavir and 3-Hydroxy-3-Methylglutaryl Coenzyme A Reductase Inhibitors 704 Atorvastatin and Simvastatin. Antimicrobial Agents and Chemotherapy vol. 45 3445-3450 (2001). 705 48. Longo, J. et al. The mevalonate pathway is an actionable vulnerability of $t(4 ; 14)$-positive multiple 706 myeloma. Leukemia (2020) doi:10.1038/s41375-020-0962-2.

707 49. Goss, G. D. et al. A phase I study of high-dose rosuvastatin with standard dose erlotinib in patients 
708 with advanced solid malignancies. J. Transl. Med. 14, 83 (2016).

709 50. Hus, M. et al. Thalidomide, dexamethasone and lovastatin with autologous stem cell transplantation

710 as a salvage immunomodulatory therapy in patients with relapsed and refractory multiple myeloma.

711 Ann. Hematol. 90, 1161-1166 (2011).

712 51. Knox, J. J. et al. A Phase I trial of prolonged administration of lovastatin in patients with recurrent or

713 metastatic squamous cell carcinoma of the head and neck or of the cervix. Eur. J. Cancer 41,

$714 \quad 523-530(2005)$.

715 52. Kornblau, S. M. et al. Blockade of adaptive defensive changes in cholesterol uptake and synthesis in

716 AML by the addition of pravastatin to idarubicin + high-dose Ara-C: a phase 1 study. Blood 109,

$717 \quad 2999-3006$ (2007).

718 53. Longo, J. et al. A pilot window-of-opportunity study of preoperative fluvastatin in localized prostate

719 cancer. Prostate Cancer Prostatic Dis. (2020) doi:10.1038/s41391-020-0221-7.

720 54. Murtola, T. J. et al. Atorvastatin Versus Placebo for Prostate Cancer Before Radical

721 Prostatectomy-A Randomized, Double-blind, Placebo-controlled Clinical Trial. Eur. Urol. 74,

$722 \quad$ 697-701 (2018).

723 55. El-Hachem, N. et al. Integrative Cancer Pharmacogenomics to Infer Large-Scale Drug Taxonomy.

724 Cancer Res. 77, 3057-3069 (2017).

725 56. Tanimoto, T. T. An Elementary Mathematical Theory of Classification and Prediction. (International

$726 \quad$ Business Machines Corporation, 1958).

727 57. Guha, R. \& Others. Chemical informatics functionality in R. J. Stat. Softw. 18, 1-16 (2007).

728 58. Smirnov, P. et al. PharmacoGx: an R package for analysis of large pharmacogenomic datasets.

729 Bioinformatics 32, 1244-1246 (2016).

730 59. Subramanian, T. \& Others. Molecular Signatures Database (MSigDB). Proc. Natl. Acad. Sci. U. S. A.

$731 \quad 102,15545-15550(2005)$

732 60. Romero, P. et al. Computational prediction of human metabolic pathways from the complete human

733 genome. Genome Biol. 6, R2 (2005).

734 61. Kanehisa, M. \& Goto, S. KEGG: kyoto encyclopedia of genes and genomes. Nucleic Acids Res. 28, 
$735 \quad 27-30(2000)$.

736 62. Du, J. et al. KEGG-PATH: Kyoto encyclopedia of genes and genomes-based pathway analysis using 737 a path analysis model. Mol. Biosyst. 10, 2441-2447 (2014).

738 63. Caspi, R. et al. The MetaCyc database of metabolic pathways and enzymes and the BioCyc 739 collection of pathway/genome databases. Nucleic Acids Res. 44, D471-80 (2016).

740 64. Wang, B. et al. Similarity network fusion for aggregating data types on a genomic scale. Nat.

$741 \quad$ Methods 11, 333-337 (2014).

742 65. Csardi, G., Nepusz, T. \& Others. The igraph software package for complex network research.

743 InterJournal, complex systems 1695, 1-9 (2006).

744 66. Bray, N. L., Pimentel, H., Melsted, P. \& Pachter, L. Near-optimal probabilistic RNA-seq quantification.

$745 \quad$ Nat. Biotechnol. 34, 525-527 (2016).

746 67. Frankish, A. et al. GENCODE reference annotation for the human and mouse genomes. Nucleic $747 \quad$ Acids Res. 47, D766-D773 (2019).

748 68. Wirapati, P. et al. Meta-analysis of gene expression profiles in breast cancer: toward a unified 749 understanding of breast cancer subtyping and prognosis signatures. Breast Cancer Res. 10, R65 $750 \quad$ (2008).

751 69. Gendoo, D. M. A. et al. Genefu: an R/Bioconductor package for computation of gene 752 expression-based signatures in breast cancer. Bioinformatics 32, 1097-1099 (2016).

753 70. Vichai, V. \& Kirtikara, K. Sulforhodamine B colorimetric assay for cytotoxicity screening. Nat. Protoc. $754 \quad 1,1112-1116(2006)$.

755 71. Subramanian, A. et al. Gene set enrichment analysis: a knowledge-based approach for interpreting 756 genome-wide expression profiles. Proc. Natl. Acad. Sci. U. S. A. 102, 15545-15550 (2005).

757 72. Liberzon, A. et al. Molecular signatures database (MSigDB) 3.0. Bioinformatics 27, 1739-1740 758 (2011).

759 73. Varemo, L., Nielsen, J. \& Nookaew, I. Enriching the gene set analysis of genome-wide data by 760 incorporating directionality of gene expression and combining statistical hypotheses and methods.

$761 \quad$ Nucleic Acids Res. 41, 4378-4391 (2013). 
762 74. Ashburner, M. et al. Gene ontology: tool for the unification of biology. The Gene Ontology

763 Consortium. Nat. Genet. 25, 25-29 (2000).

764 75. Sarri6, D. et al. Epithelial-mesenchymal transition in breast cancer relates to the basal-like

765 phenotype. Cancer Res. 68, 989-997 (2008). 


\section{FIGURE 1}

A

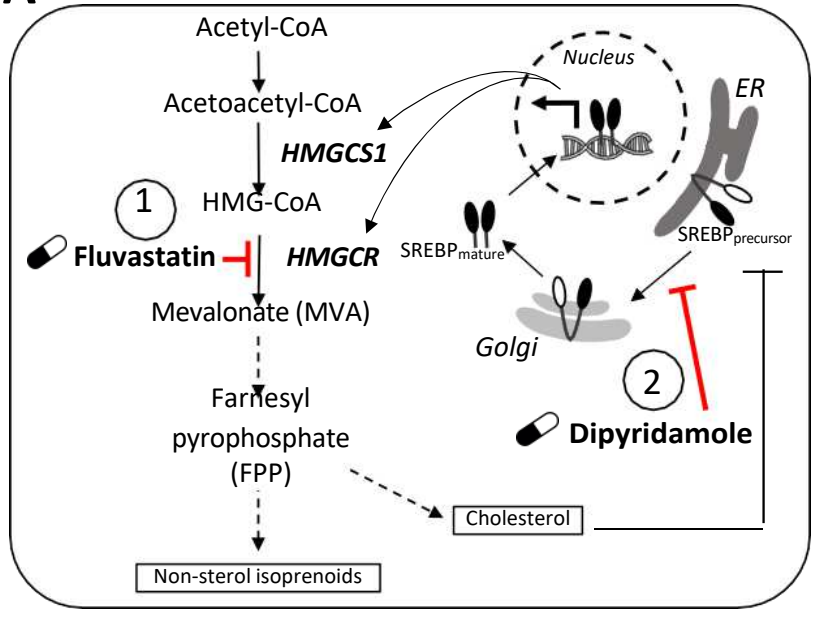

C Structure Sensitivity $\bigcirc$ Perturbation

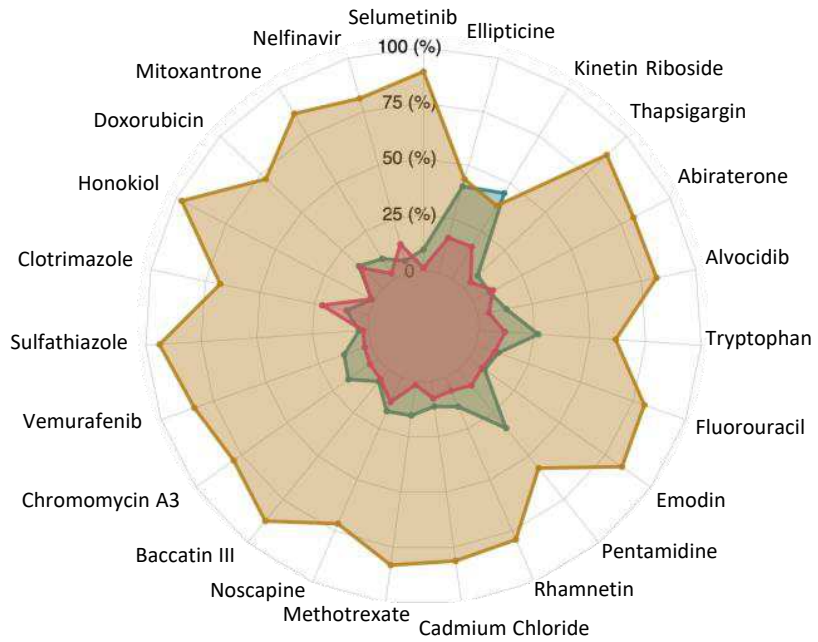

B

\section{Computational Screen}

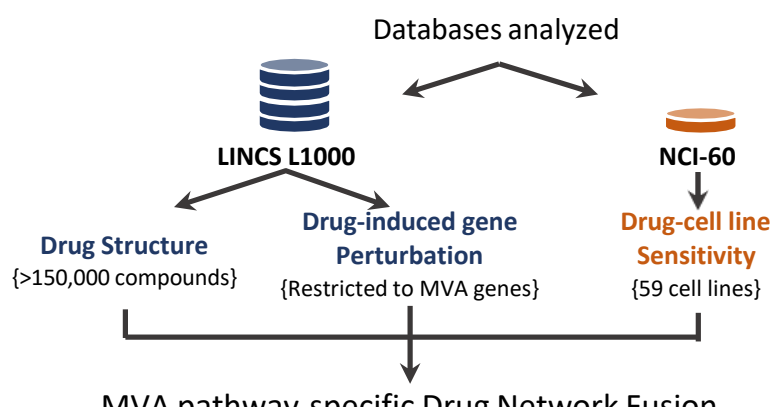

MVA pathway-specific Drug Network Fusion

(MVA-DNF)

Permutation test

$\downarrow$

EMODIN

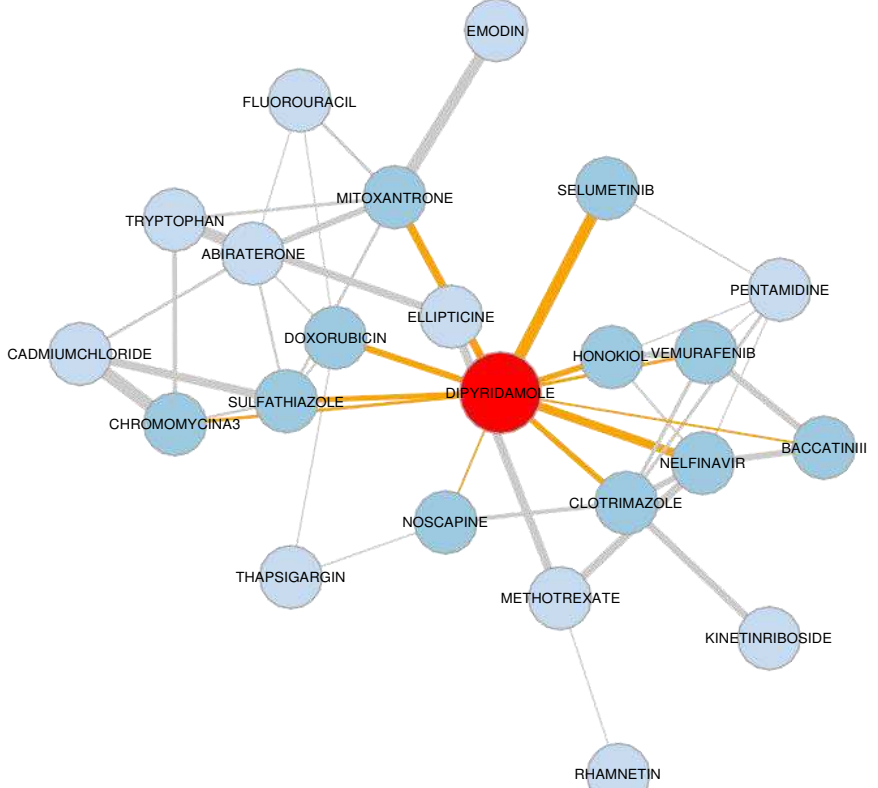




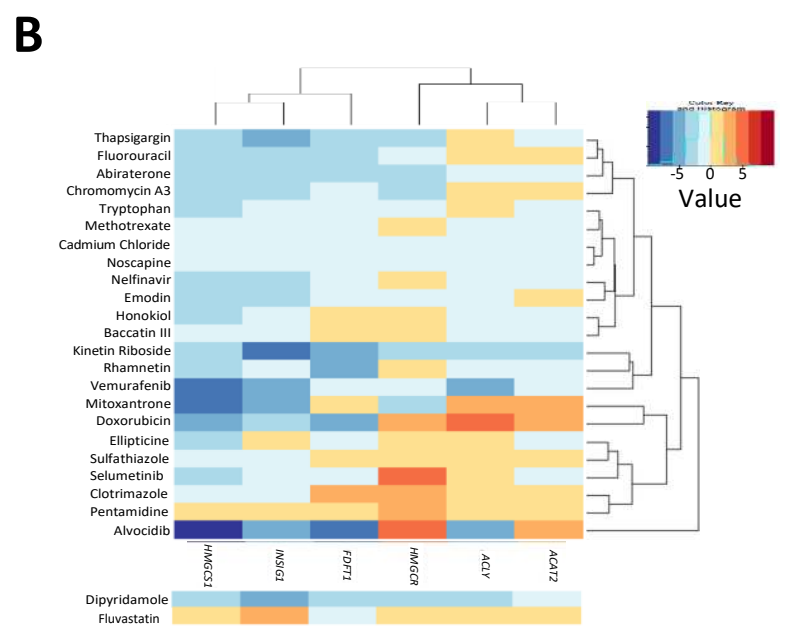

C

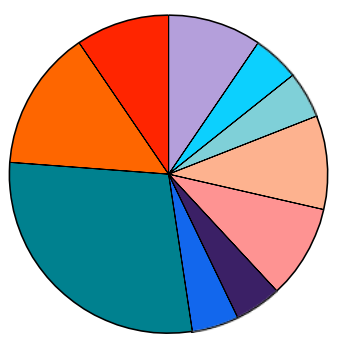

Total $=21$

\section{SUPP FIGURE 1}

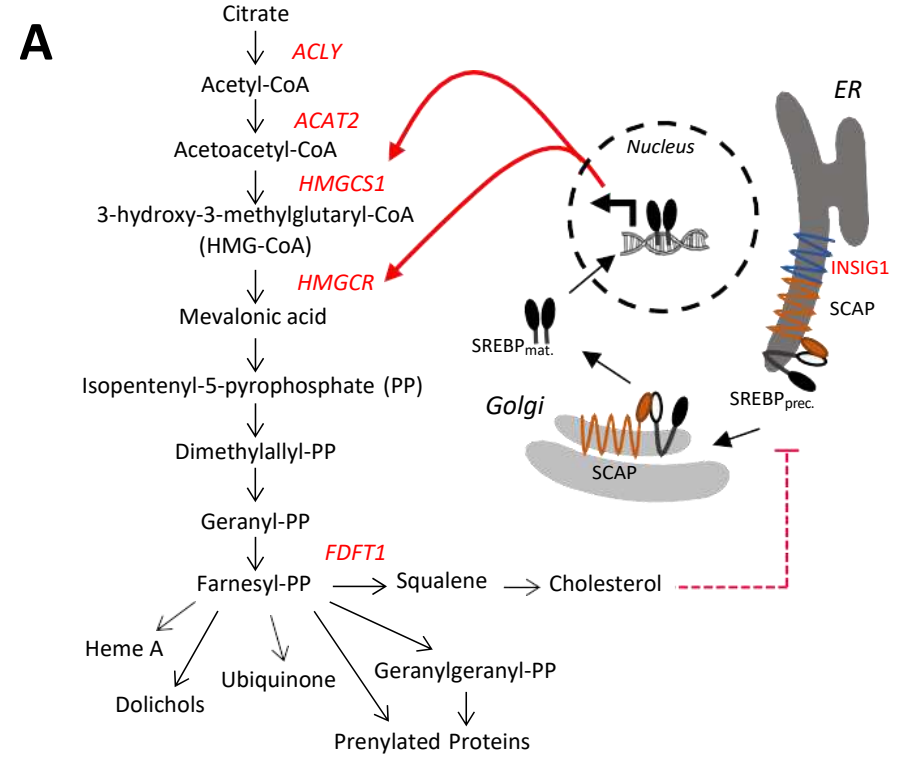

A

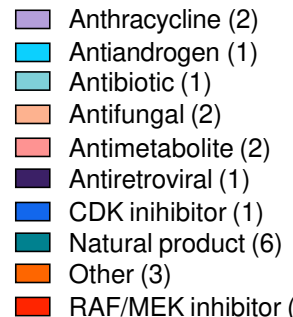

Anthracycline (2)

Antiandrogen (1)

(1)

Antifungal (2)

Antimetabolite (2)

Antiretroviral (1)

CDK inihibitor (1)

$\square$ Other (3)

RAF/MEK inhibitor (2)

Total=21 


\section{SUPP FIGURE 2}

A
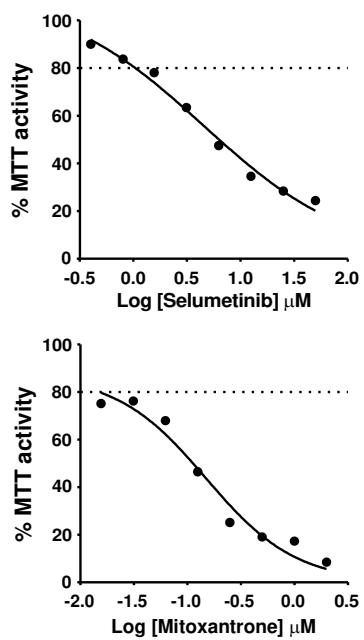

MDA-MB-231
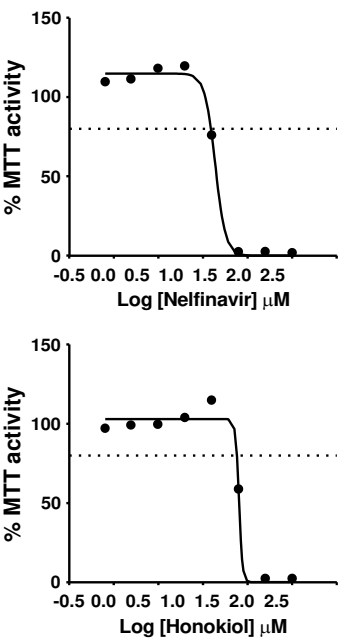

B

HCC1937
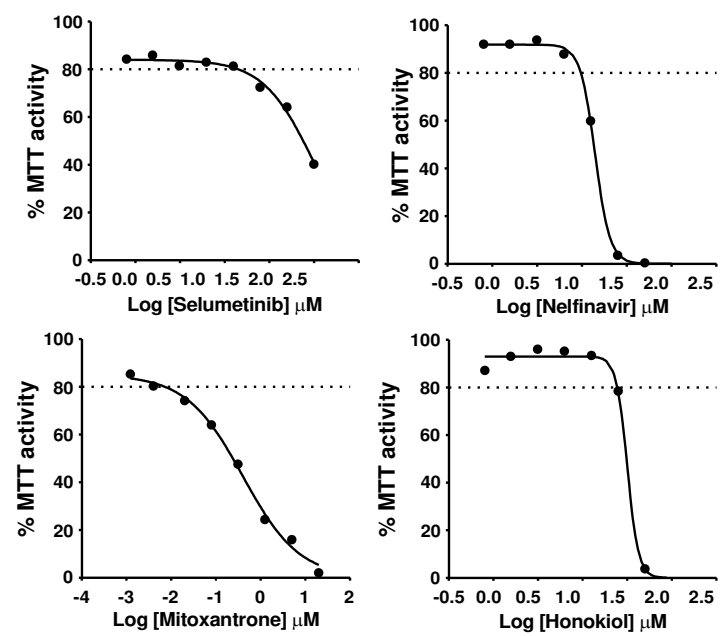

C

\begin{tabular}{|c|c|c|c|c|}
\hline & \multicolumn{2}{|c|}{ MDA-MB-231 } & \multicolumn{2}{c|}{ HCC1937 } \\
\hline Drug & Dose & Interpolated MTT activity & Dose & Interpolated MTT activity \\
\hline Selumetinib & $0.4 \mu \mathrm{M}$ & $91.92 \%$ & $1 \mu \mathrm{M}$ & $83.97 \%$ \\
\hline Nelfinavir & $3 \mu \mathrm{M}$ & $114.8 \%$ & $3 \mu \mathrm{M}$ & $91.8 \%$ \\
\hline Mitoxantrone & $0.01 \mu \mathrm{M}$ & $\sim 80 \%$ & $0.001 \mu \mathrm{M}$ & $85.4 \%$ \\
\hline Honokiol & $12 \mu \mathrm{M}$ & $102.99 \%$ & $10 \mu \mathrm{M}$ & $92.97 \%$ \\
\hline
\end{tabular}



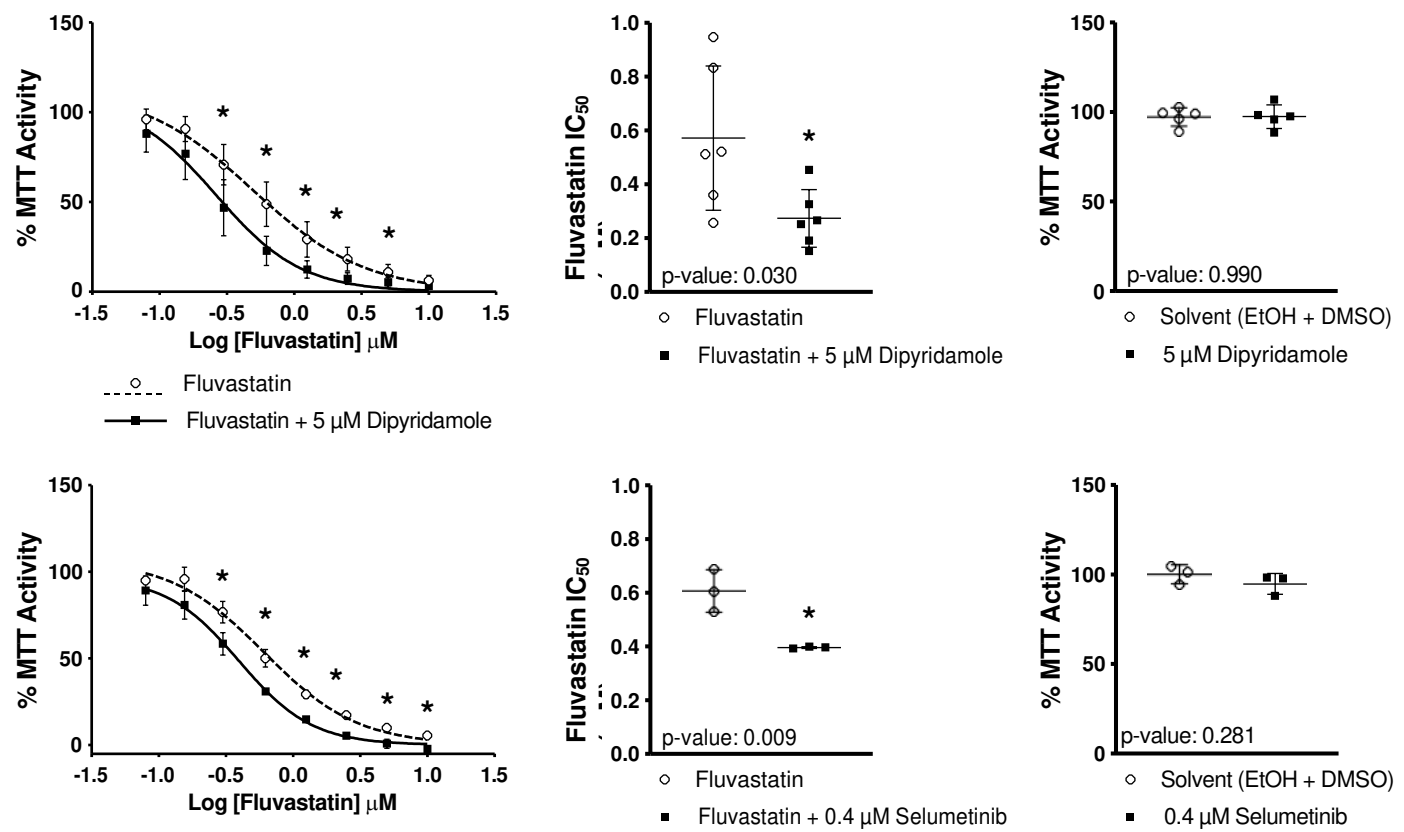

_. Fluvastatin

$\longrightarrow$ Fluvastatin $+0.4 \mu \mathrm{M}$ Selumetinib
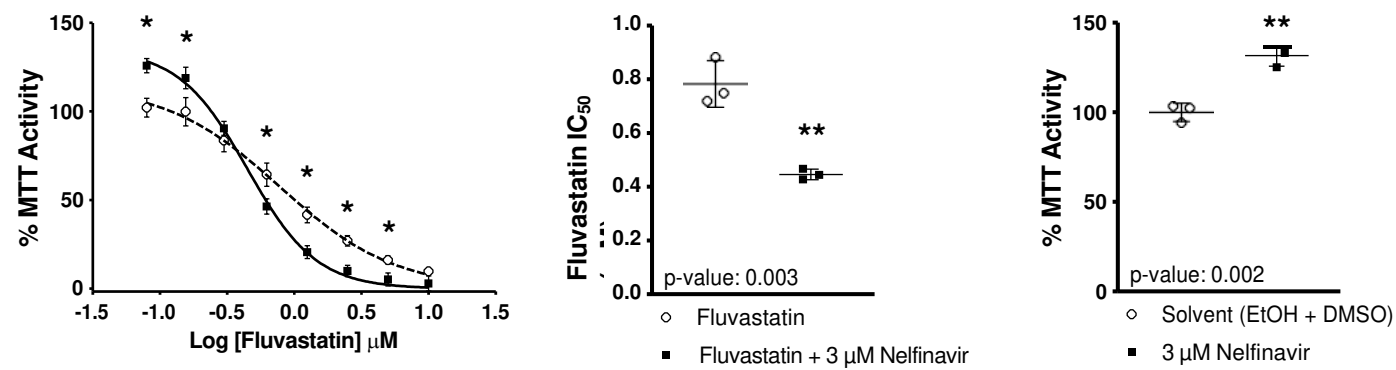

‥ Fluvastatin

$\longrightarrow$ Fluvastatin $+3 \mu \mathrm{M}$ Nelfinavir
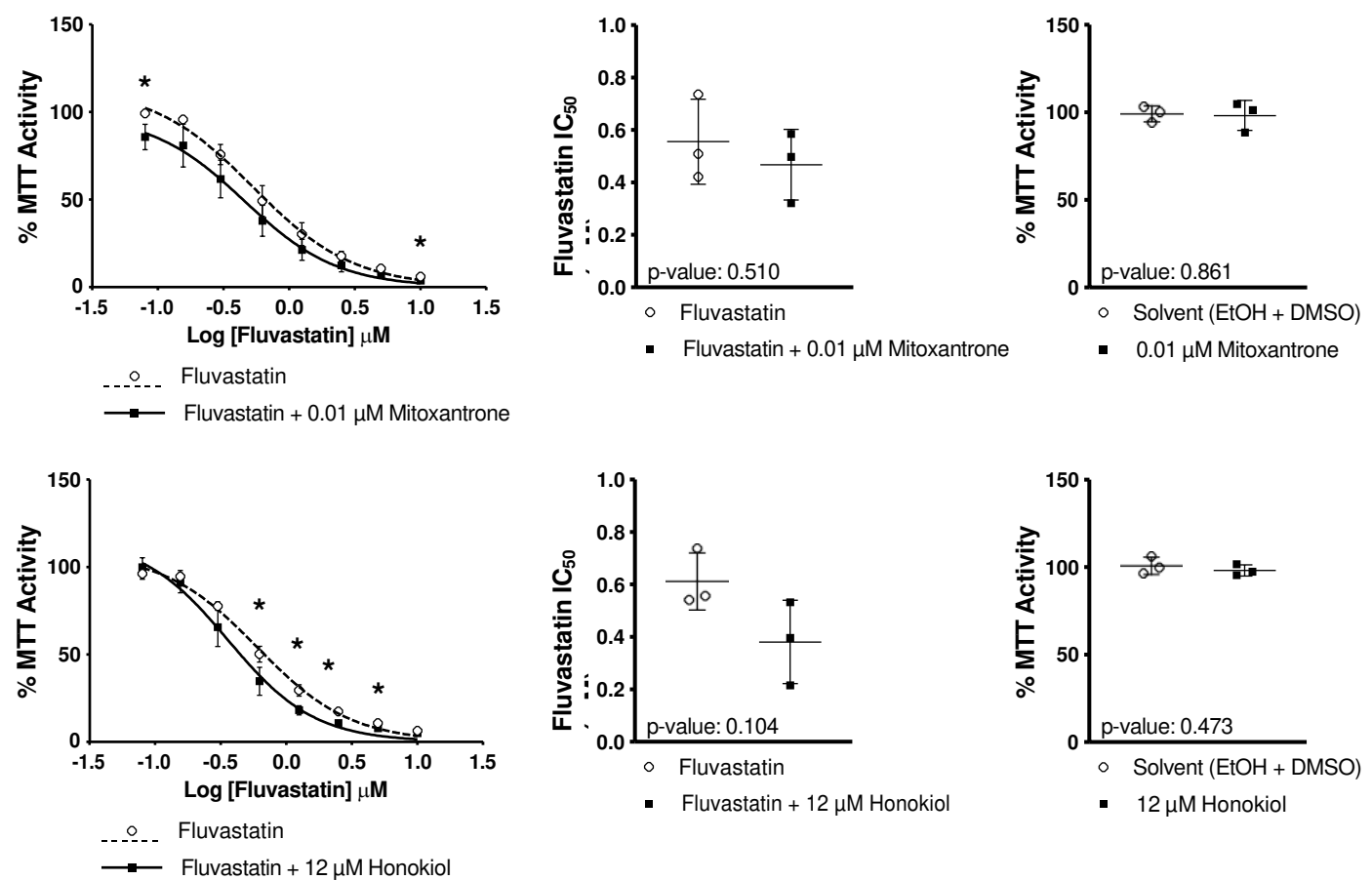


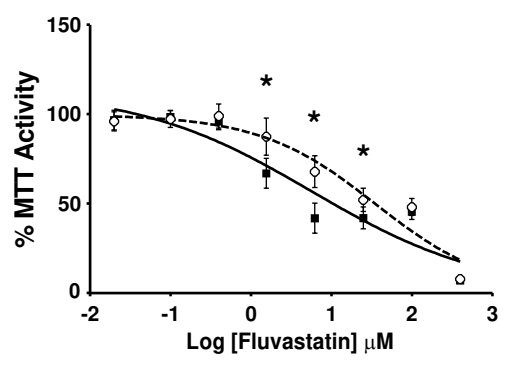

_... Fluvastatin

$\longrightarrow$ Fluvastatin $+5 \mu \mathrm{M}$ Dipyridamole

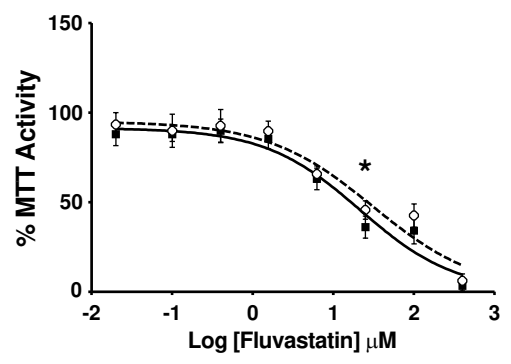

_. - Fluvastatin

$\longrightarrow$ Fluvastatin $+1 \mu \mathrm{M}$ Selumetinib

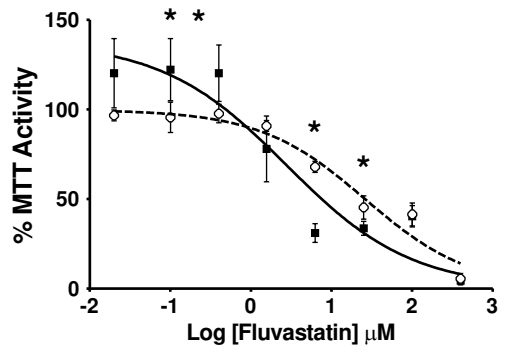

_. Fluvastatin

$\longrightarrow$ Fluvastatin $+3 \mu \mathrm{M}$ Nelfinavir

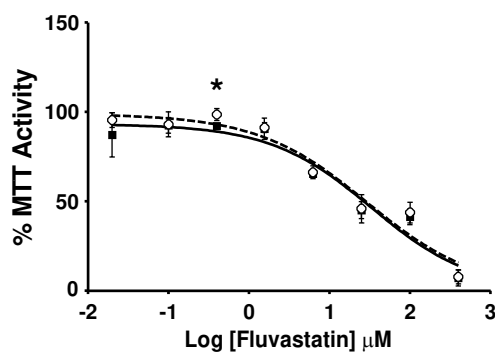

- Fluvastatin

$\longrightarrow$ Fluvastatin $+0.001 \mu \mathrm{M}$ Mitoxantrone

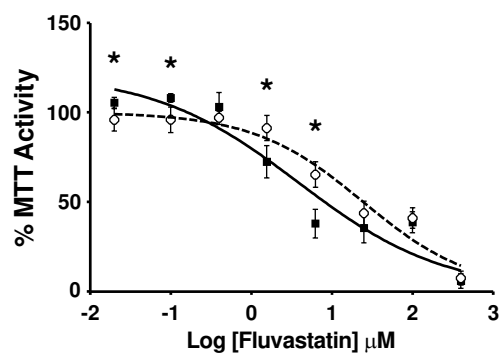

.... Fluvastatin

$\longrightarrow$ Fluvastatin $+10 \mu \mathrm{M}$ Honokiol
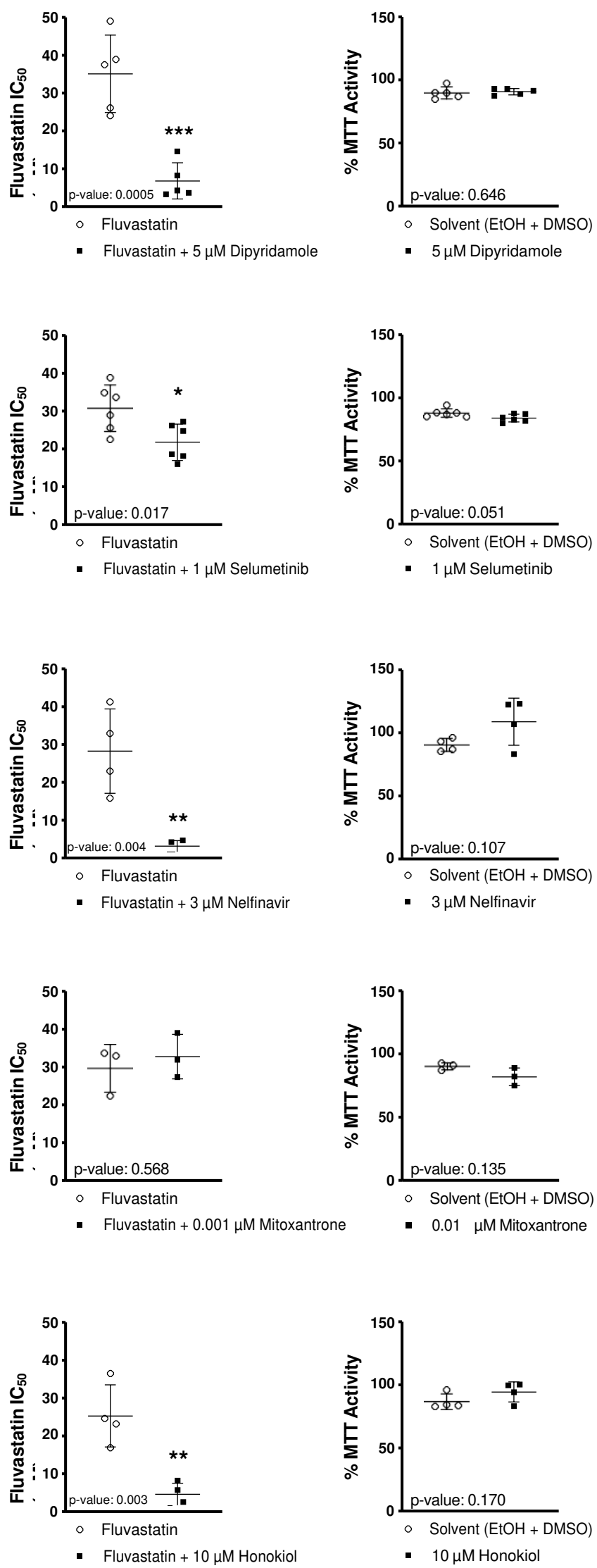
A

B MDA-MB-231

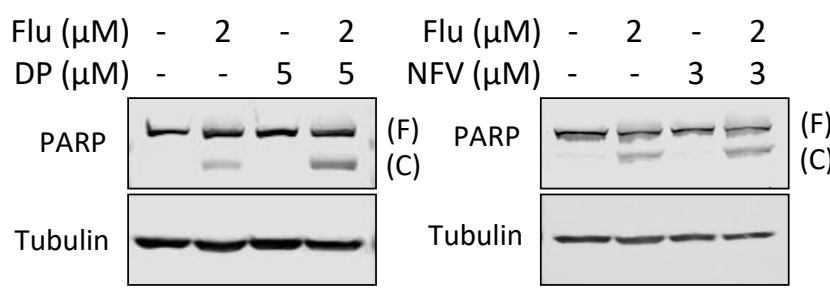

Flu $(\mu \mathrm{M})-2-2$ Flu $(\mu \mathrm{M})-2-2$ HN

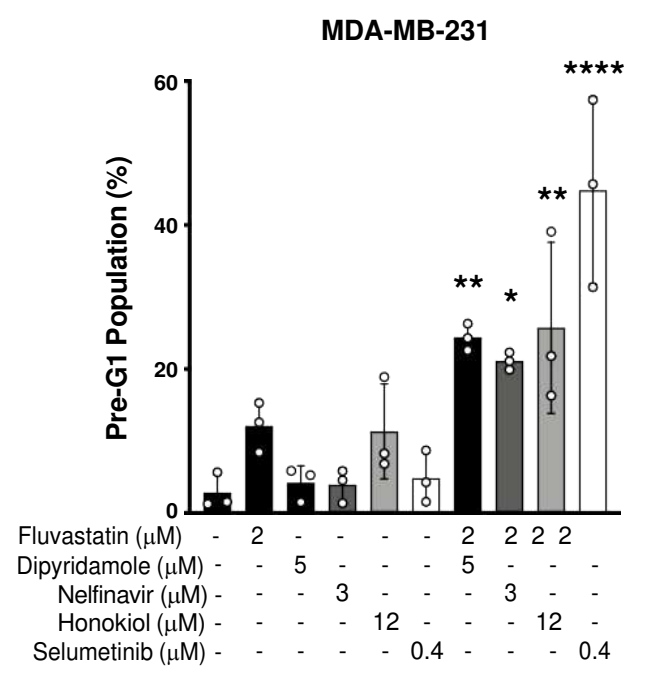

MDA-MB-231

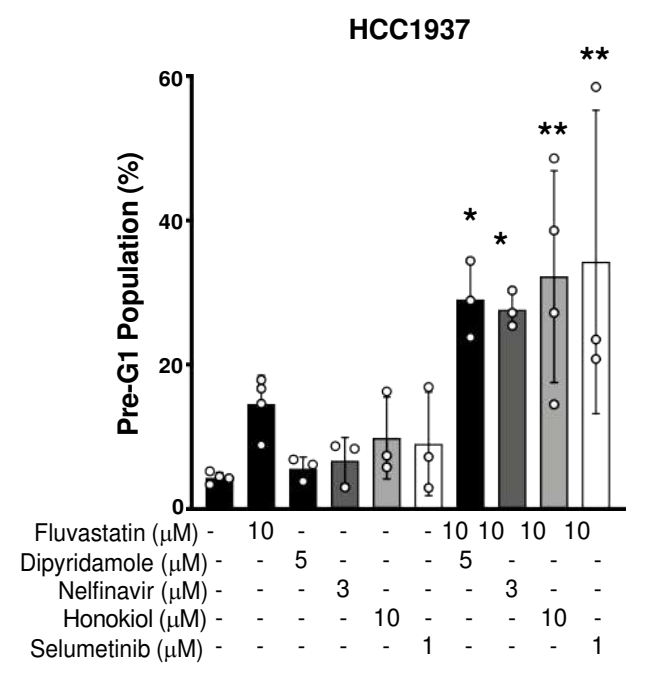

\section{HCC1937}

Flu $(\mu \mathrm{M})-10-10 \quad$ Flu $(\mu \mathrm{M})-10-10$ $\mathrm{DP}(\mu \mathrm{M})$ - $-55 \mathrm{NFV}(\mu \mathrm{M})$ - 53

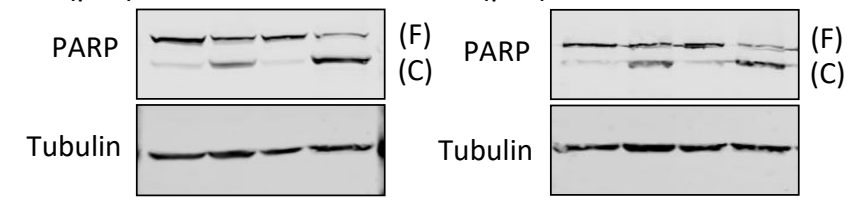

Flu $(\mu \mathrm{M})-10-10 \quad$ Flu $(\mu \mathrm{M})-10-10$ $\operatorname{HNK}(\mu \mathrm{M})$ - $\quad$ - 1010 Selu $(\mu \mathrm{M})$ - $\quad$ - 111
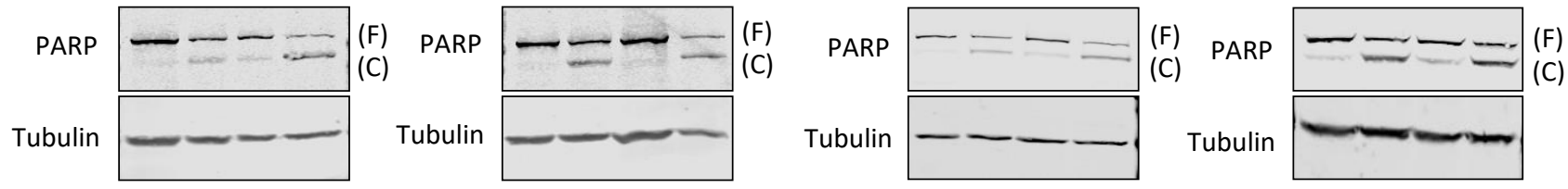

C

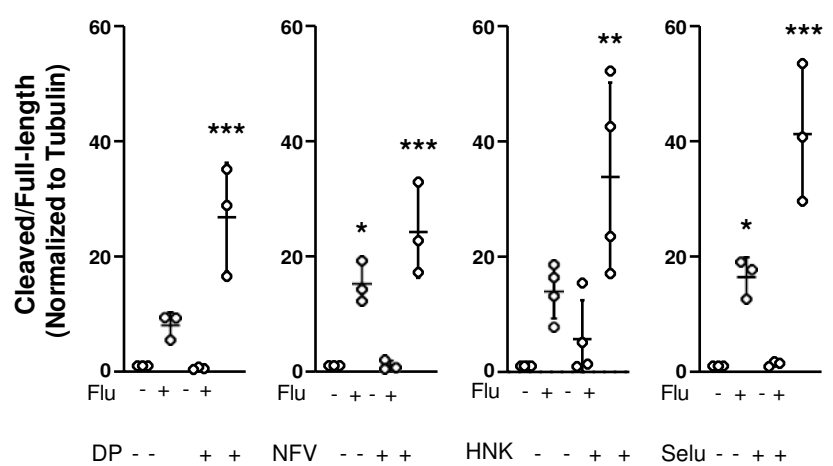

MDA-MB-231

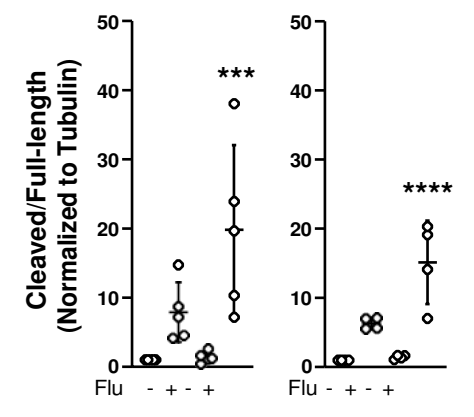

DP - - + + NFV - + + HNK - + +

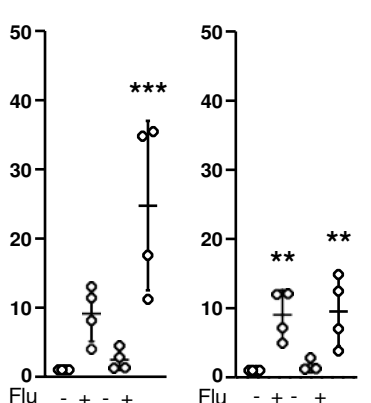

Selu - - + + 
A

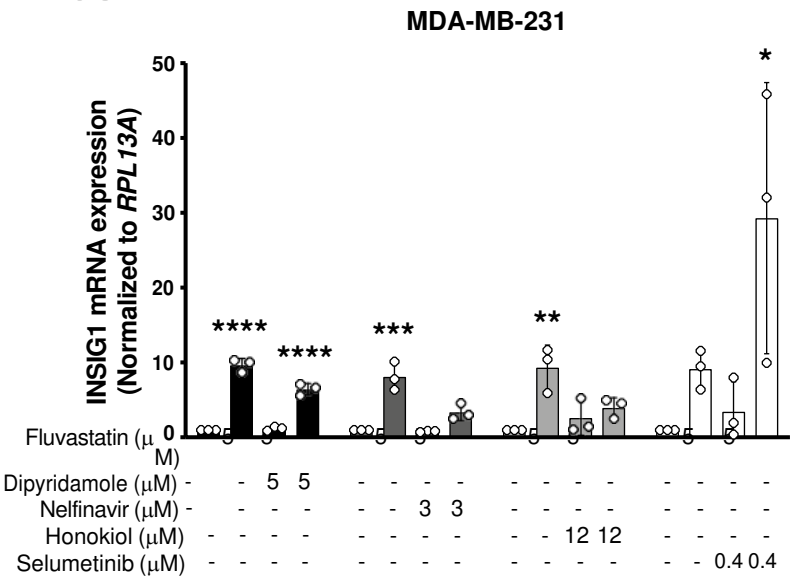

HCC1937

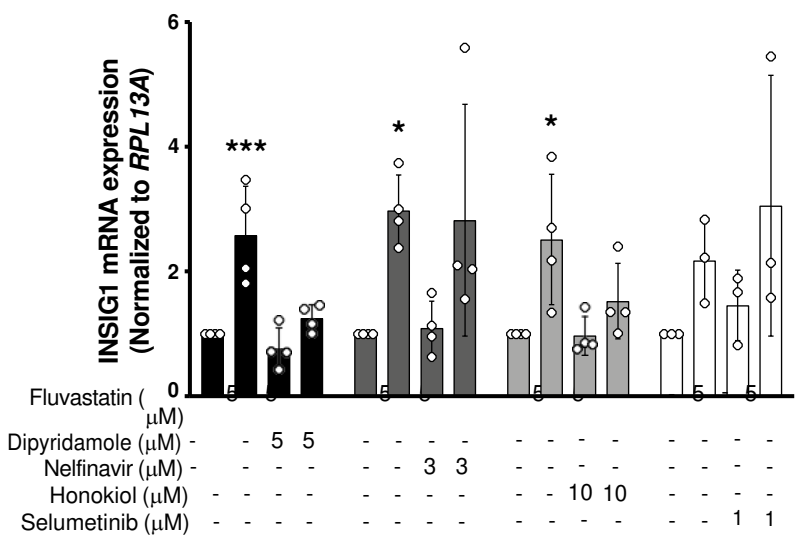

B

HCC1937

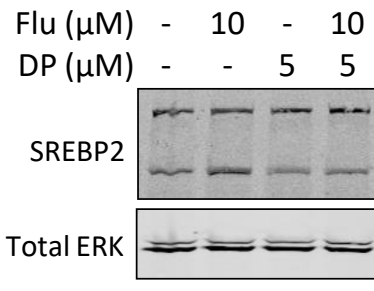

Flu $(\mu \mathrm{M})-10-10$ $\mathrm{HNK}(\mu \mathrm{M})$ - $\quad 1212$

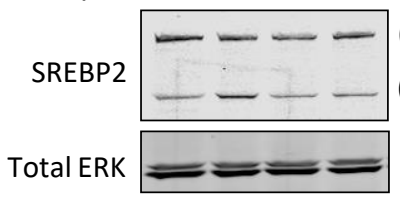

Flu $(\mu \mathrm{M})-10-10$ $N F V(\mu \mathrm{M})-\quad 33$ (P)

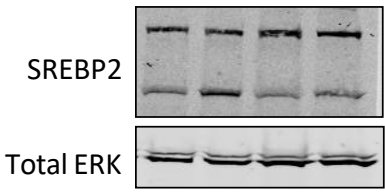

Flu $(\mu \mathrm{M})-10-10$ $\mathrm{DP}(\mu \mathrm{M})$ - $\quad 5 \quad 5$

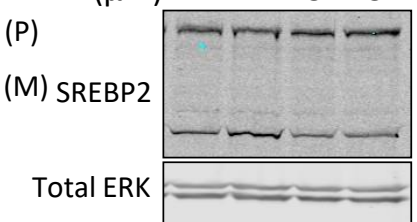

Flu $(\mu \mathrm{M})-10-10$ $\mathrm{NFV}(\mu \mathrm{M})-\quad-33$

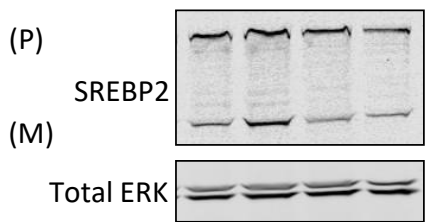

Flu $(\mu \mathrm{M})-10-10 \quad$ Flu $(\mu \mathrm{M})-10-10$ Selu $(\mu \mathrm{M}) \quad-\quad-\quad 0.40 .4$ SREBP2 Total ERK

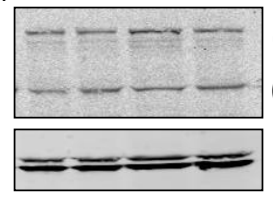

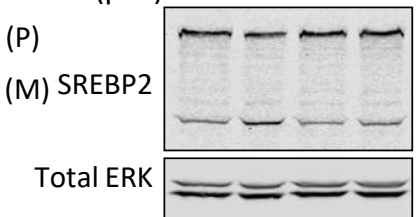

Flu $(\mu \mathrm{M})-10-10$

Selu $(\mu \mathrm{M})$ - $\quad-\quad 1 \quad 1$ (P)

SREBP2 (M)

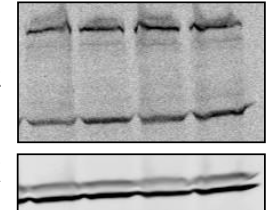

C

MDA-MB-231
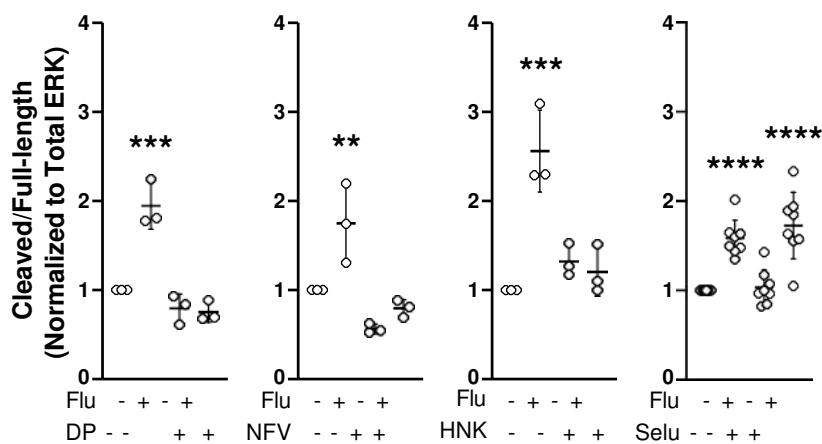

\section{HCC1937}

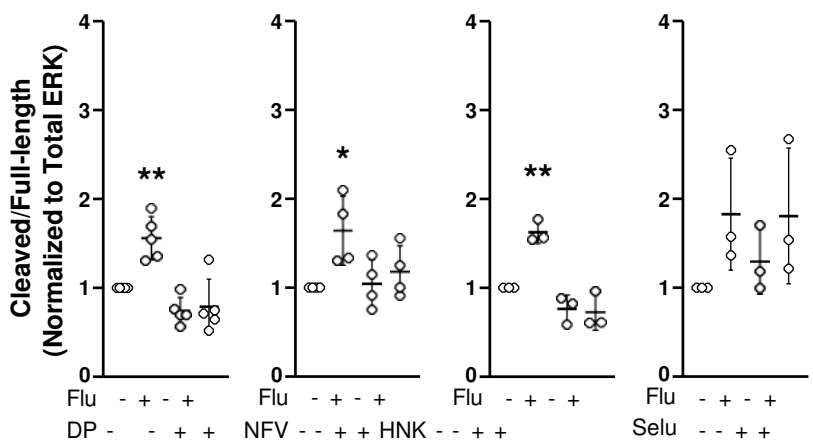




\section{SUPP FIGURE 5}

A

MDA-MB-231
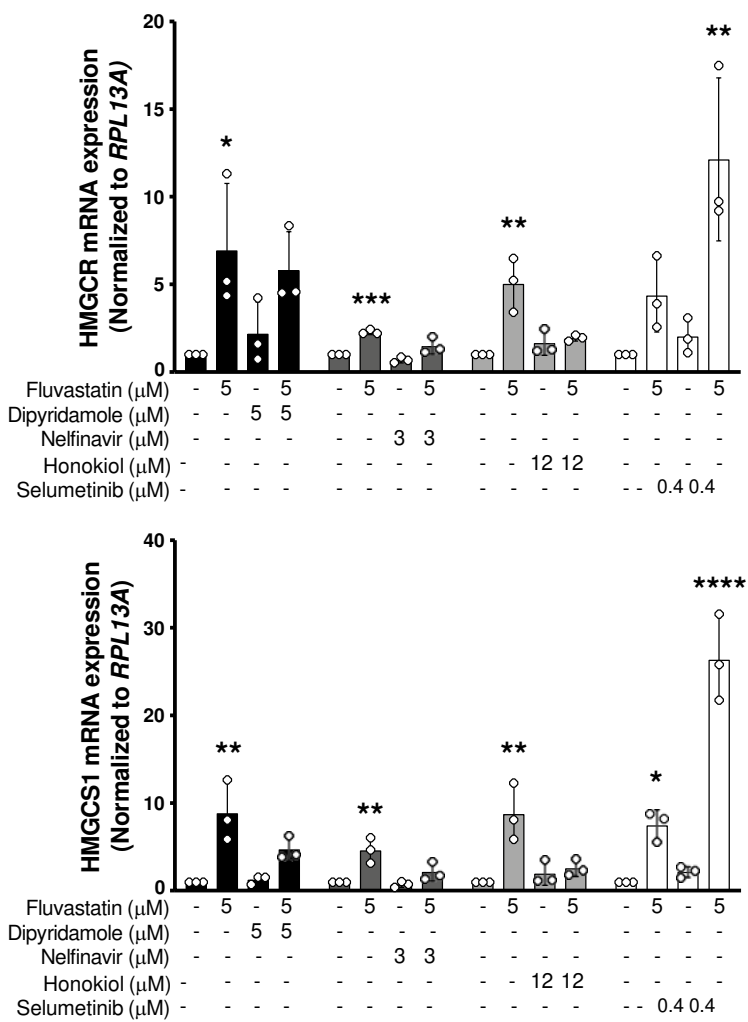

B

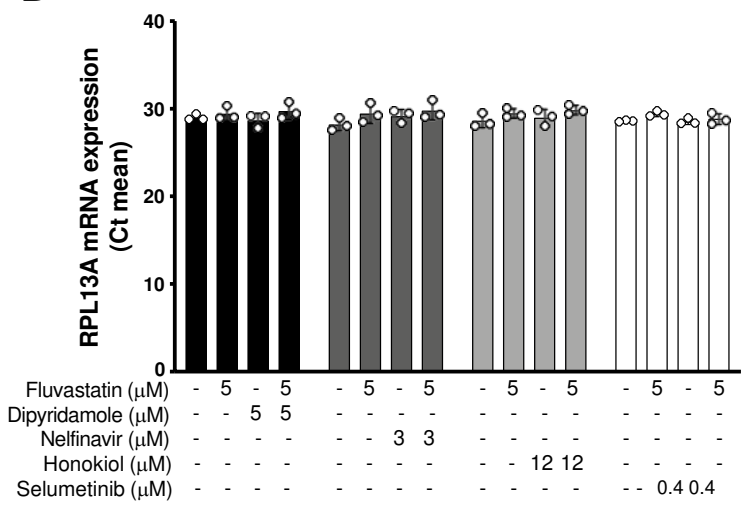


SUPP FIG 6

A
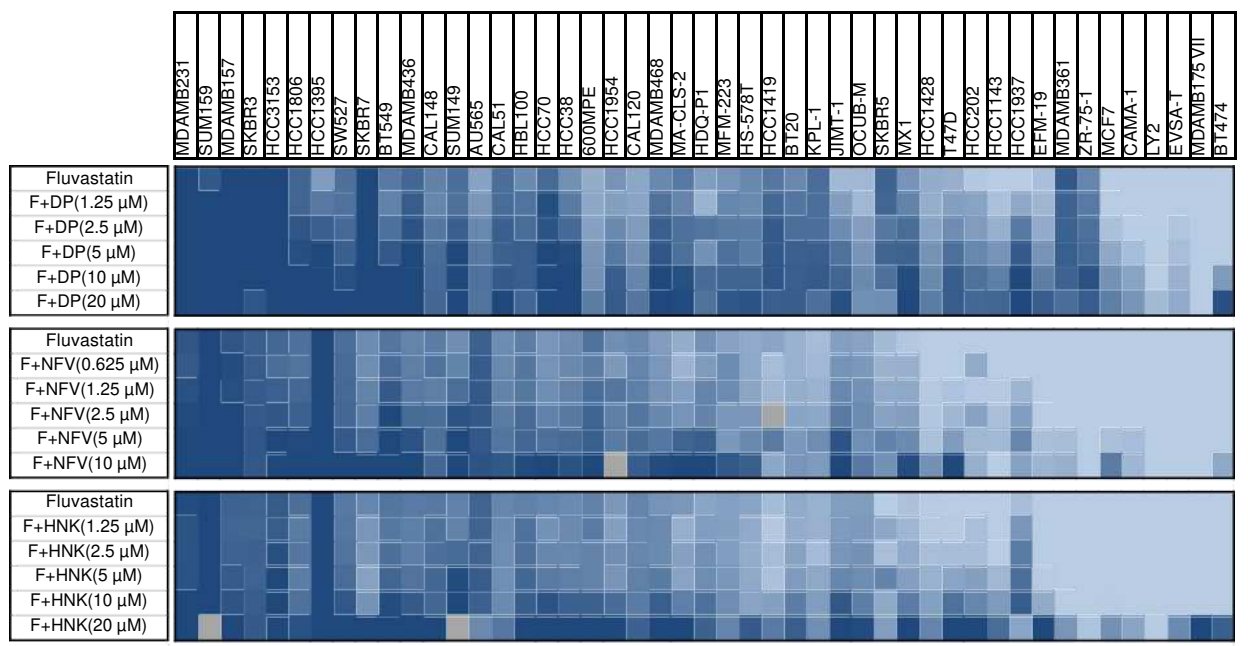

B

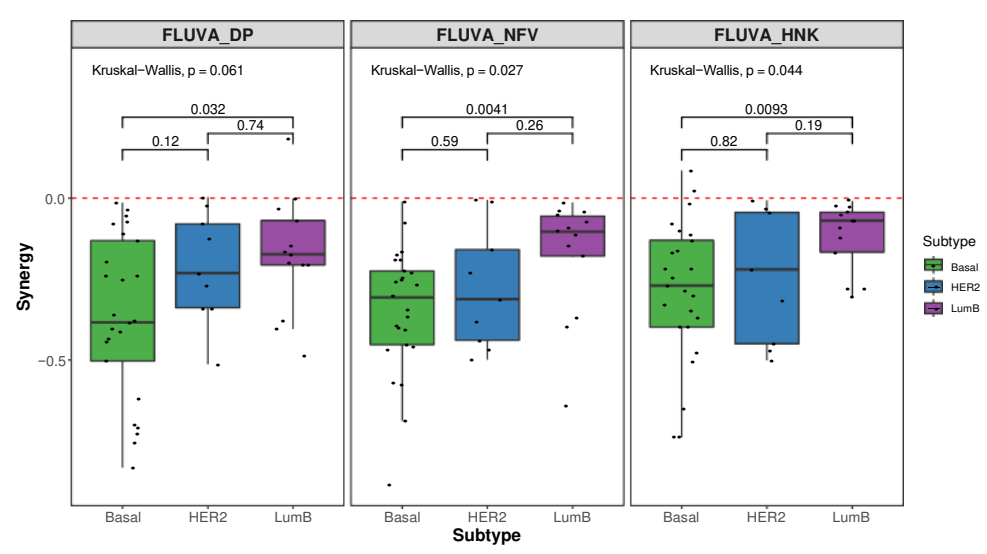

C
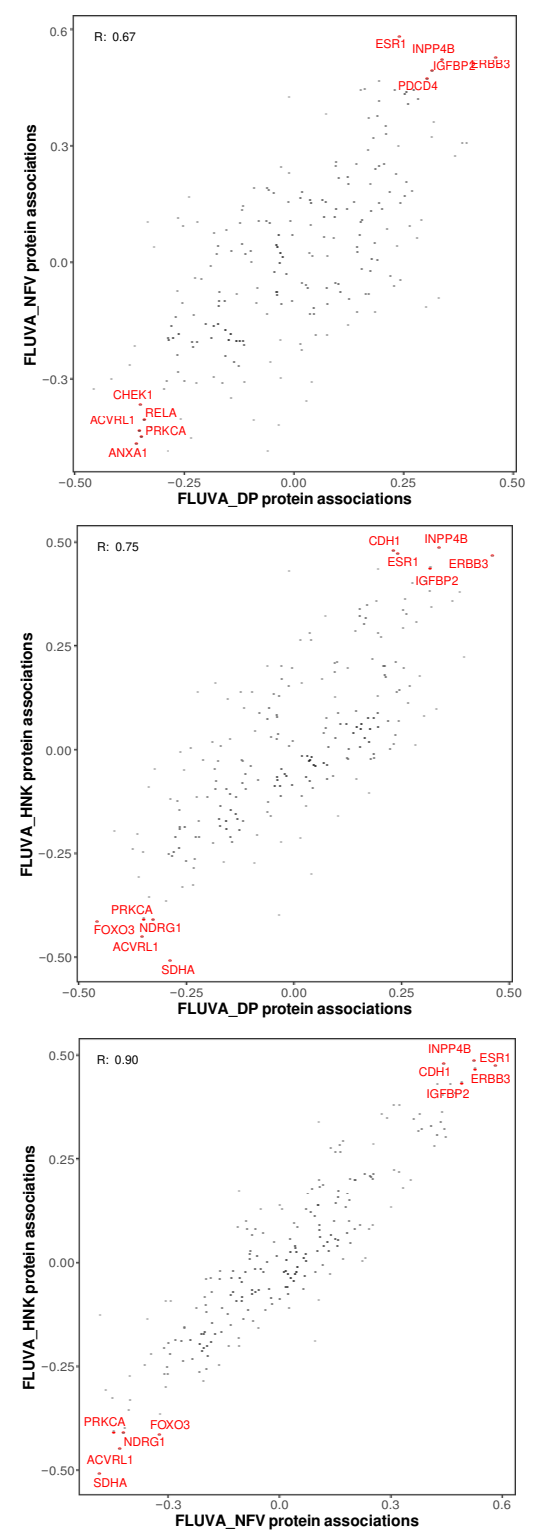

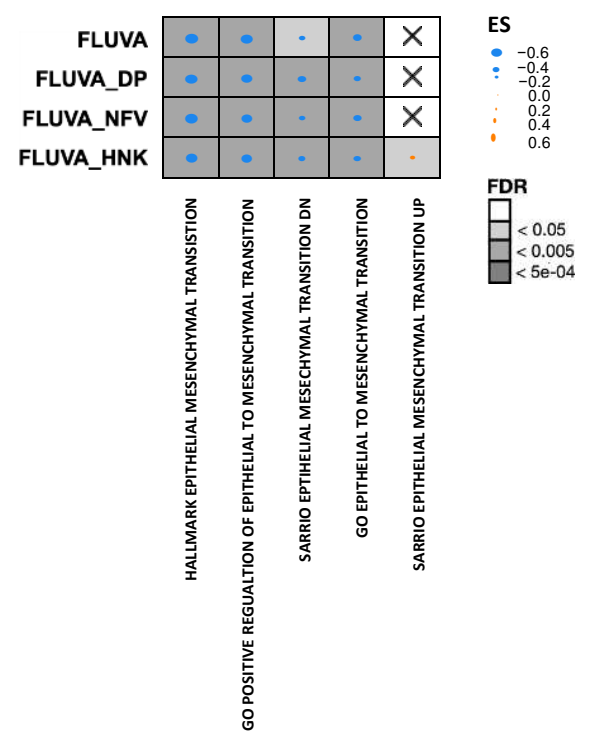

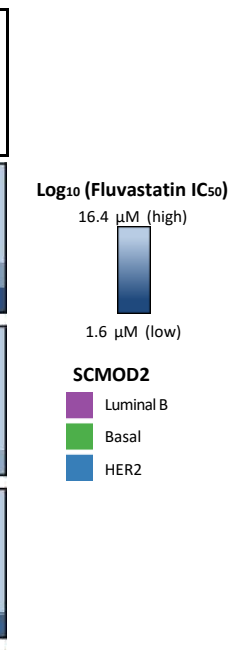

D 
A

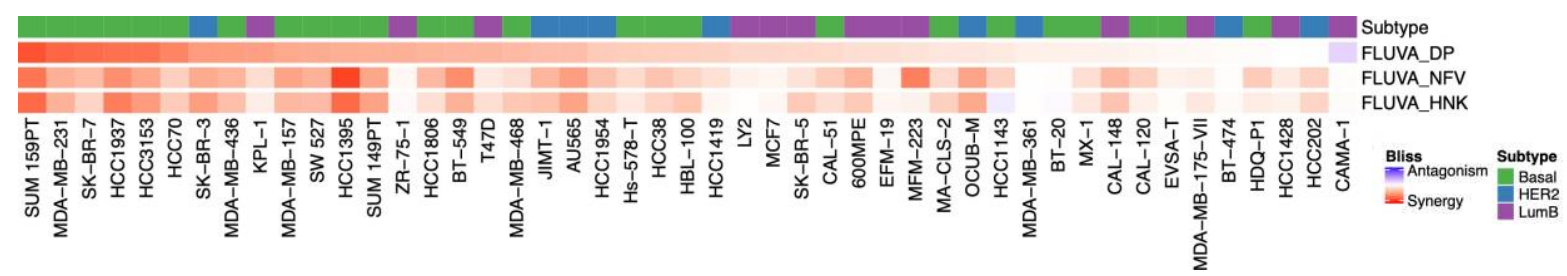

B

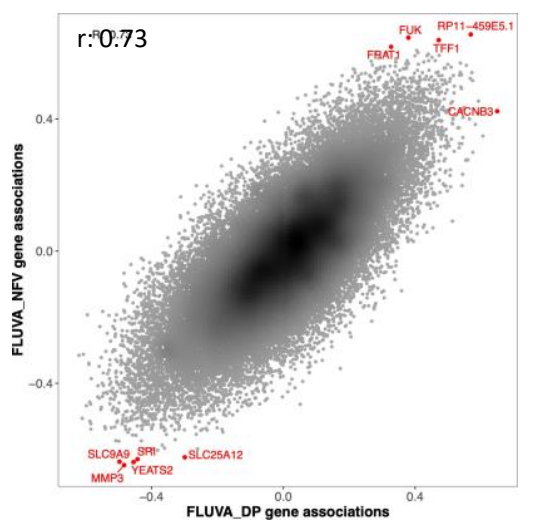

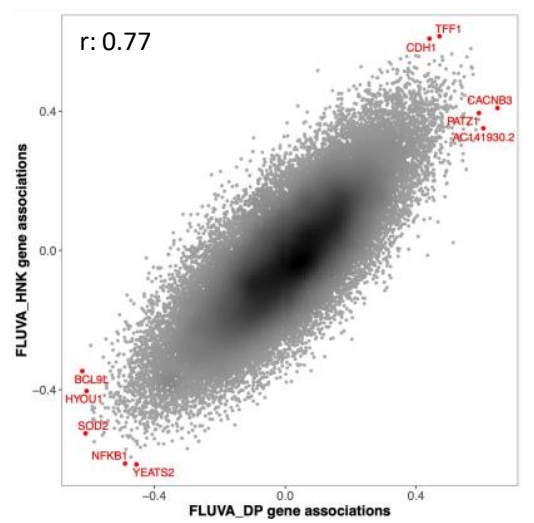

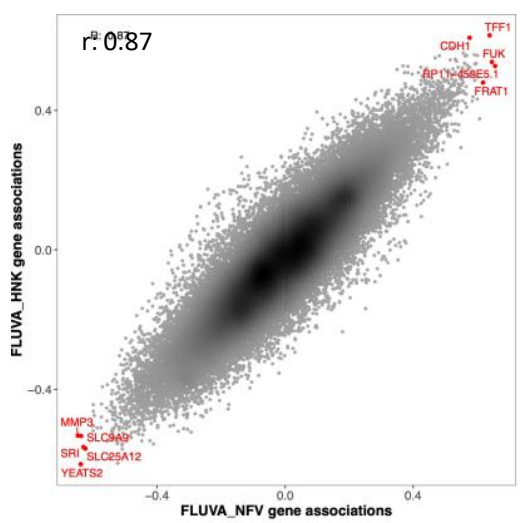

C
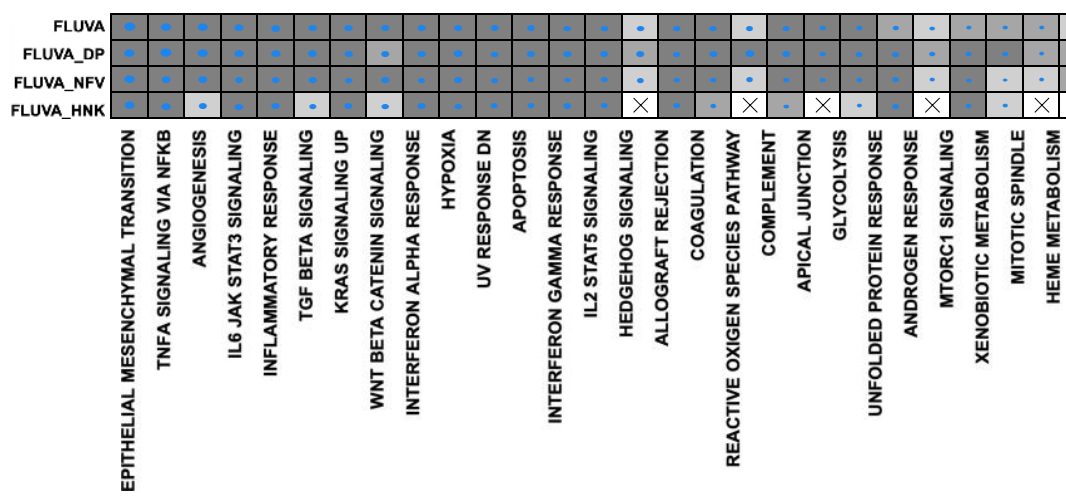

ES

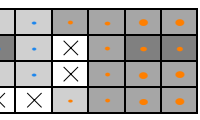

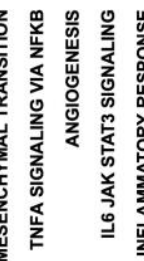
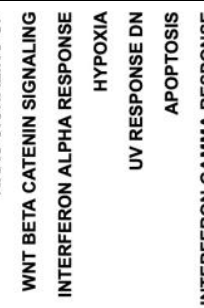

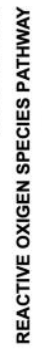
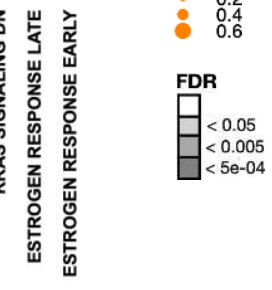

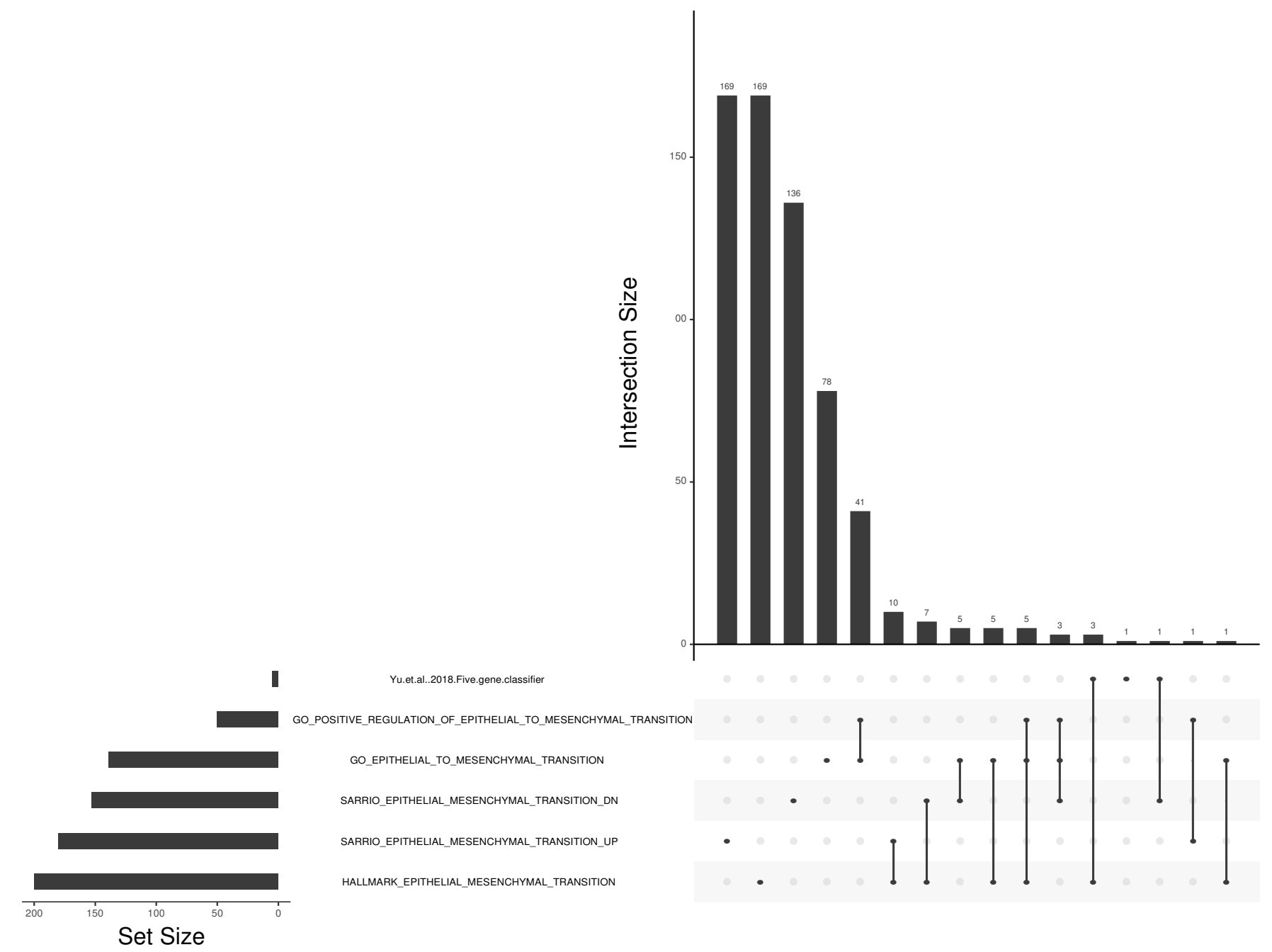

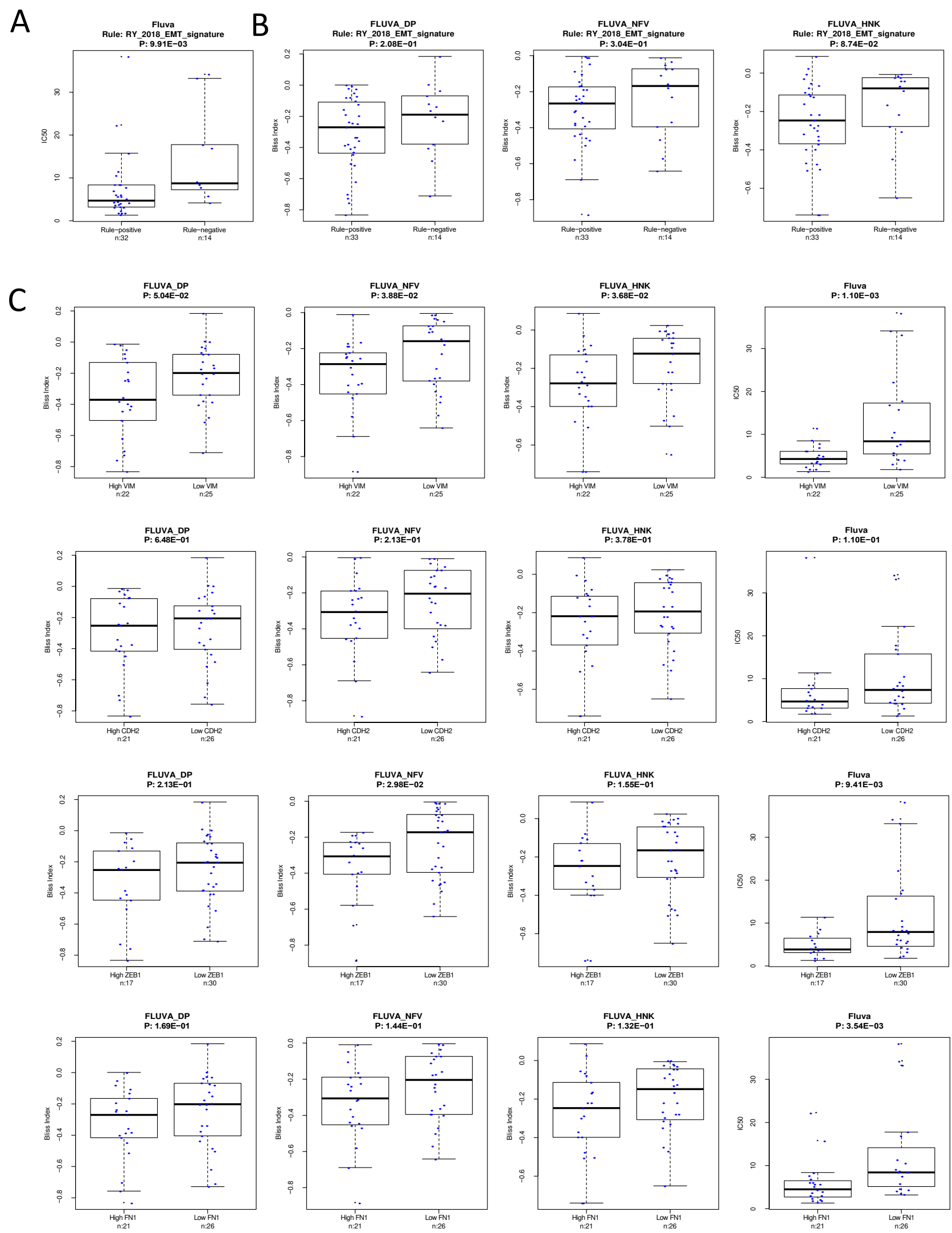
A

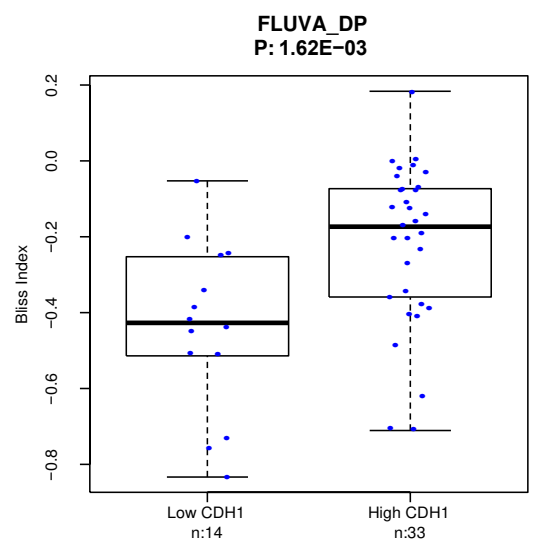

B

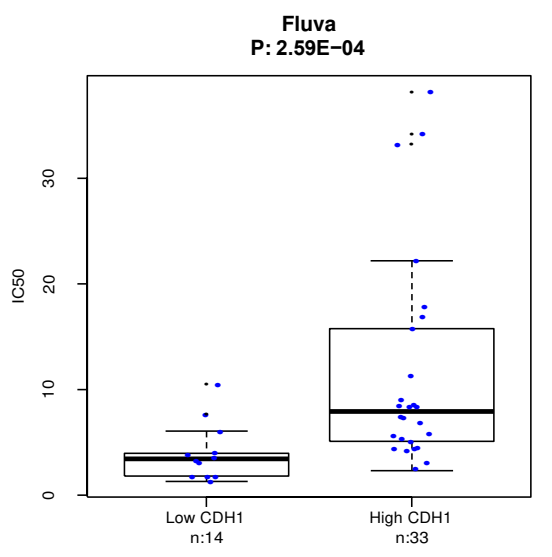

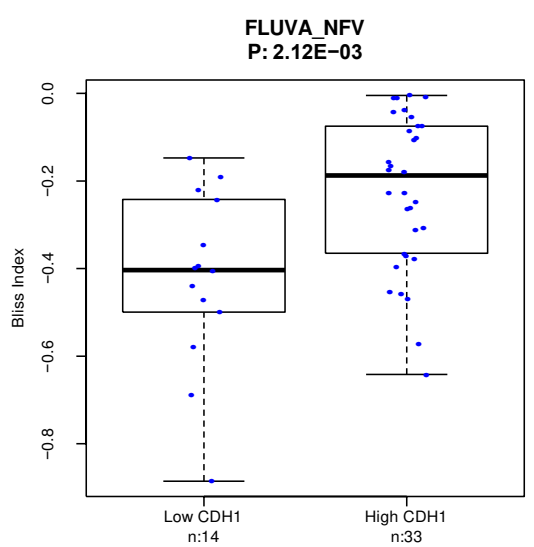

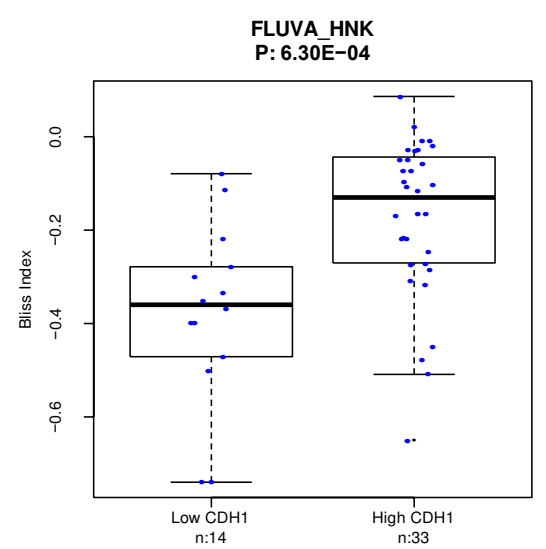

C

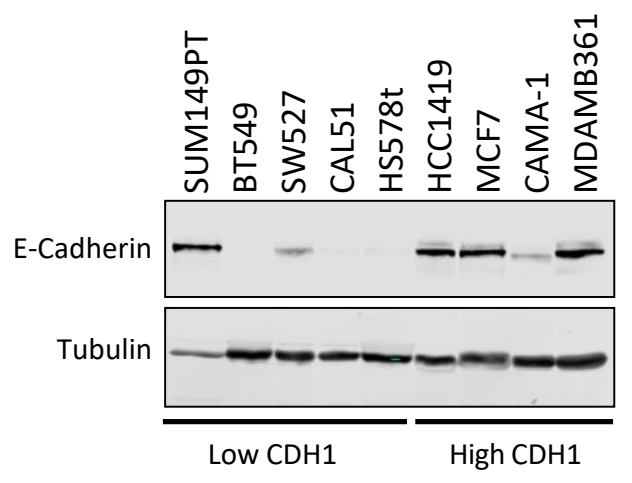

D
Protein expression (Normalized to tubulin)

E

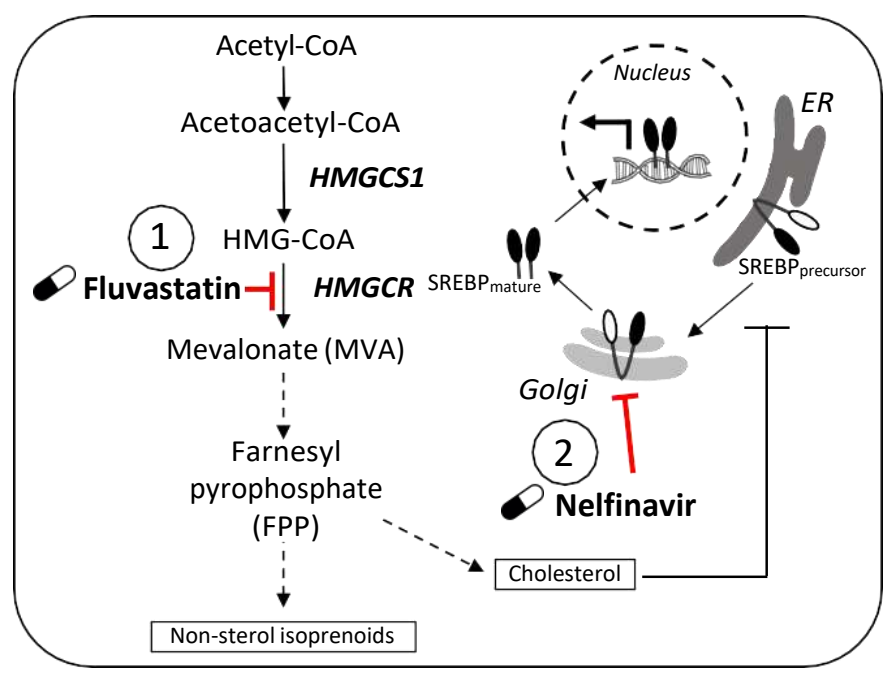




\begin{tabular}{|c|c|c|c|c|c|c|c|c|c|}
\hline \multirow[b]{2}{*}{ Hits } & \multicolumn{2}{|c|}{ Permutation Test } & \multirow{2}{*}{ 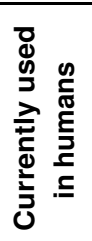 } & \multirow{2}{*}{ 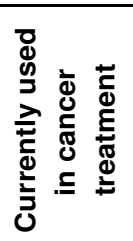 } & \multirow{2}{*}{ 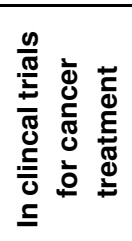 } & \multirow{2}{*}{ 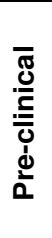 } & \multirow{2}{*}{ 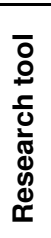 } & \multirow{2}{*}{$\begin{array}{l}\frac{0}{0} \\
\text { 릉 } \\
\text { ய }\end{array}$} & \multirow{2}{*}{$\begin{array}{l}\frac{}{0} \\
\frac{0}{0} \\
\frac{0}{0} \\
\frac{0}{0} \\
\frac{0}{0}\end{array}$} \\
\hline & $\begin{array}{l}\text { ○ } \\
\text { O } \\
\text { N }\end{array}$ & $\begin{array}{l}\frac{0}{\partial} \\
\frac{0}{\pi} \\
\text { 1' }\end{array}$ & & & & & & & \\
\hline SELUMETINIB & -3.57 & 1.77E-04 & + & & + & & & & RAF/MEK inhibitor \\
\hline NELFINAVIR & -3.28 & $5.18 \mathrm{E}-04$ & + & & + & & & & Antiretroviral \\
\hline MITOXANTRONE & -3.20 & $6.82 \mathrm{E}-04$ & + & + & + & & & & Anthracycline \\
\hline DOXORUBICIN & -3.03 & $1.22 \mathrm{E}-03$ & + & + & + & & & & Anthracycline \\
\hline HONOKIOL & -2.96 & $1.53 \mathrm{E}-03$ & & & & + & & & Natural product \\
\hline CLOTRIMAZOLE & -2.95 & $1.57 \mathrm{E}-03$ & + & & & & & & Antifungal \\
\hline SULFATHIAZOLE & -2.89 & $1.90 \mathrm{E}-03$ & & & & + & & & Antibiotic \\
\hline VEMURAFENIB & -2.67 & $3.79 E-03$ & + & + & + & & & & RAF/MEK inhibitor \\
\hline CHROMOMYCINA3 & -2.64 & 4.13E-03 & & & & + & + & Toxin & Antibiotic \\
\hline BACCATINIII & -2.53 & $5.69 \mathrm{E}-03$ & & & & + & & & Natural product \\
\hline NOSCAPINE & -2.51 & $6.11 \mathrm{E}-03$ & & & + & + & & & Natural product \\
\hline METHOTREXATE & -2.29 & $1.11 \mathrm{E}-02$ & + & + & + & & & & Antimetabolite \\
\hline CADMIUMCHLORIDE & -2.22 & $1.33 \mathrm{E}-02$ & & & & + & + & Carcinogen & Other \\
\hline RHAMNETIN & -2.18 & $1.45 \mathrm{E}-02$ & & & & + & & & Natural product \\
\hline PENTAMIDINE & -2.18 & $1.46 \mathrm{E}-02$ & + & & + & & & & Antifungal \\
\hline EMODIN & -2.16 & $1.55 \mathrm{E}-02$ & & & & + & & & Natural product \\
\hline FLUOROURACIL & -2.11 & $1.75 \mathrm{E}-02$ & + & + & + & & & & Antimetabolite \\
\hline TRYPTOPHAN & -1.92 & $2.76 \mathrm{E}-02$ & + & & + & & & & Other \\
\hline ALVOCIDIB & -1.82 & $3.44 \mathrm{E}-02$ & + & + & + & & & & CDK inhibitor \\
\hline ABIRATERONE & -1.77 & $3.81 \mathrm{E}-02$ & + & + & + & & & & Antiandrogen \\
\hline THAPSIGARGIN & -1.71 & $4.36 \mathrm{E}-02$ & & & & + & & & Other \\
\hline KINETINRIBOSIDE & -1.66 & $4.84 \mathrm{E}-02$ & & & & + & & & Other \\
\hline ELLIPTICINE & -1.65 & $4.92 \mathrm{E}-02$ & & & & + & & & Natural product \\
\hline
\end{tabular}




\section{Figures}

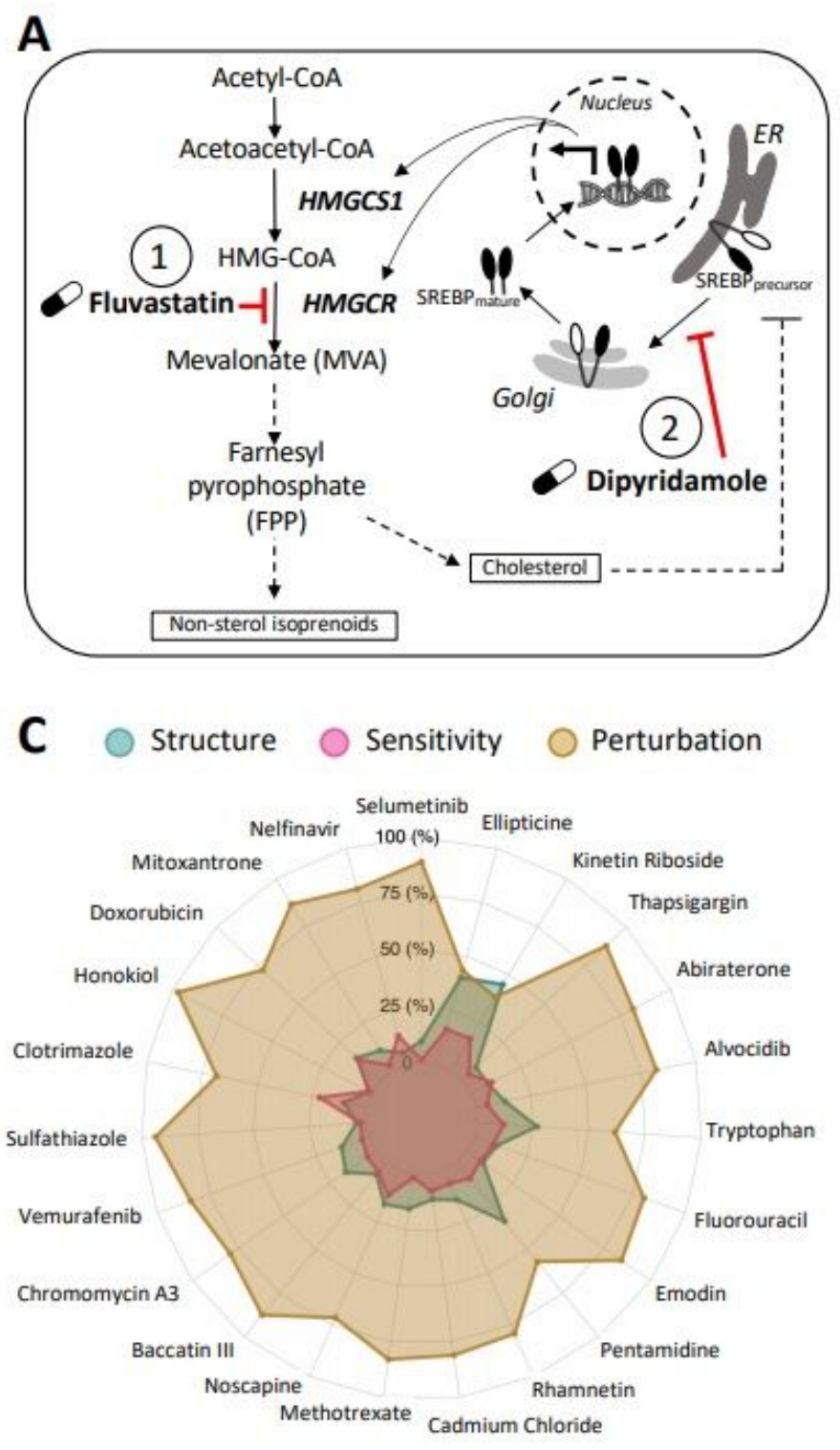

B

Computational Screen

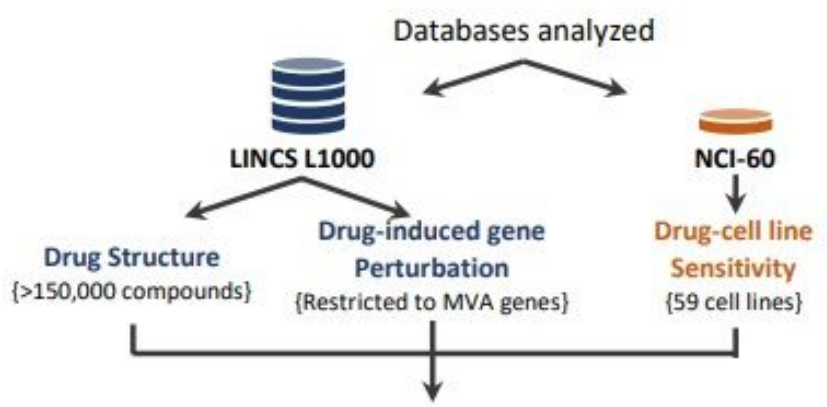

MVA pathway-specific Drug Network Fusion (MVA-DNF)

Permutation test

$\downarrow$

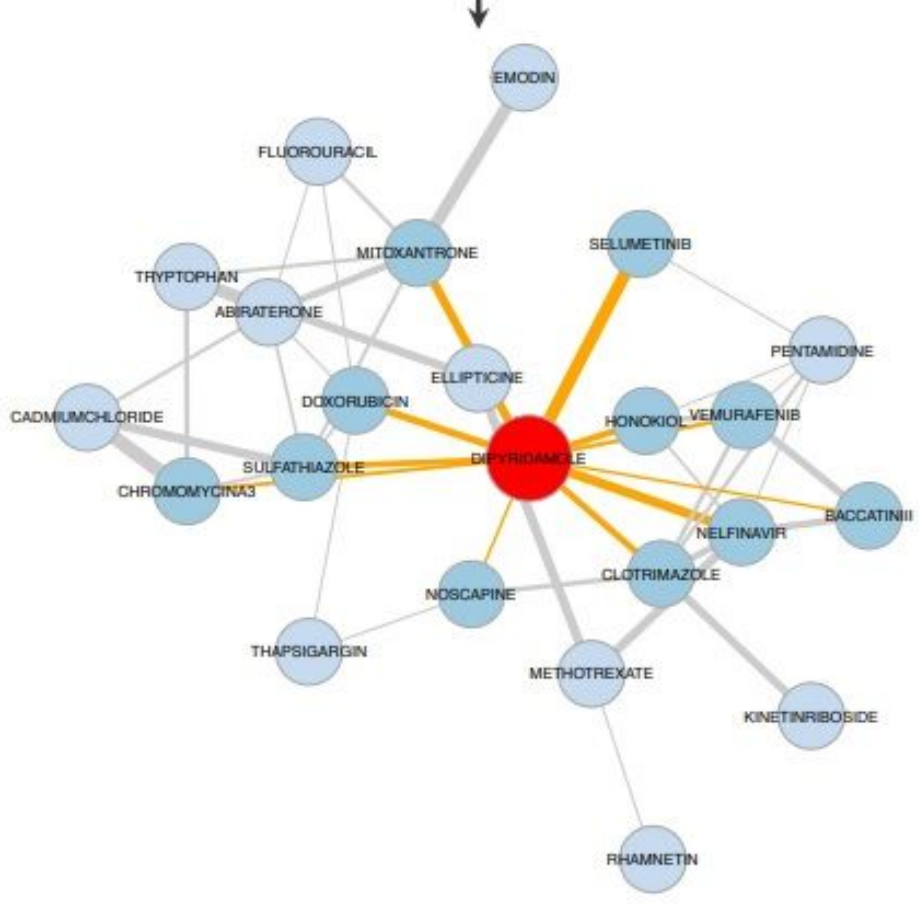

\section{Figure 1}

A schematic of the mevalonate (MVA) pathway and overview of the computational pharmacogenomics (A) In response to fluvastatin treatment (labelled with 1), MVA pathway end-product levels decrease, triggering an SREBP-mediated feedback response that activates MVA pathway-associated gene expression to restore cholesterol and other non-sterol end-product Dipyridamole (DP) (labelled with 2) blocks the SREBP-mediated feedback response, thereby potentiating fluvastatin-induced cancer cell (B) An overview of the computational pharmacogenomics workflow, MVA-DNF, used to identify the top 23 "dipyridamole-like" candidates and visualized as a compound MVA-DNF combines drug structure, drug sensitivity, and drug-induced gene perturbation datasets restricted to six MVA pathway-specific Permutation specificity testing was performed to select compounds that have a degree of specificity to 
the mevalonate pathway and Statistical significance of compounds similar to dipyridamole was assessed by comparing to 999 networks generated from random selection of six genes within the drug perturbation A network representation of dipyridamole and top 23 statistically-significant ( $p$-value $<0.05)$ "dipyridamole-like" compounds are Each node represents a compound and edges connect compounds based on statistical significance of $p$-value $<0.01$. Darker blue nodes and orange edges represent the compounds connected to dipyridamole, and edge thickness represents the associated $p$-value between the (C) Radar plot of the top 23 dipyridamole-like compounds ( $p$-value $<0.05$ ), where the contribution of each individual layer of the MVA-DNF (drug structure, sensitivity, and perturbation) is Percent contribution of each layer is shown from the center $(0 \%)$ to the outer edges $(100 \%)$. 
A

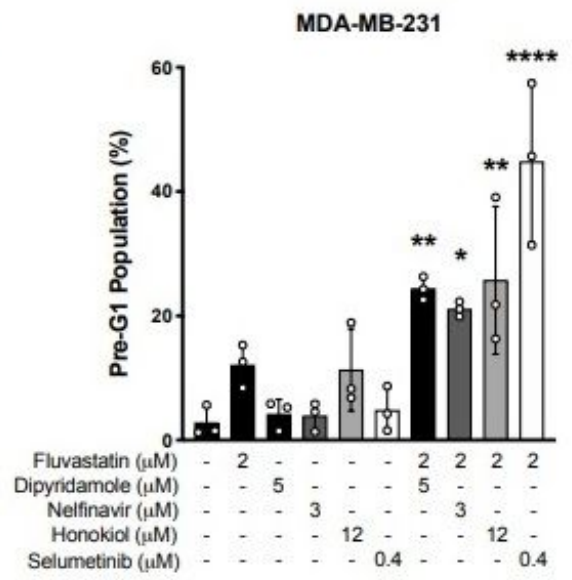

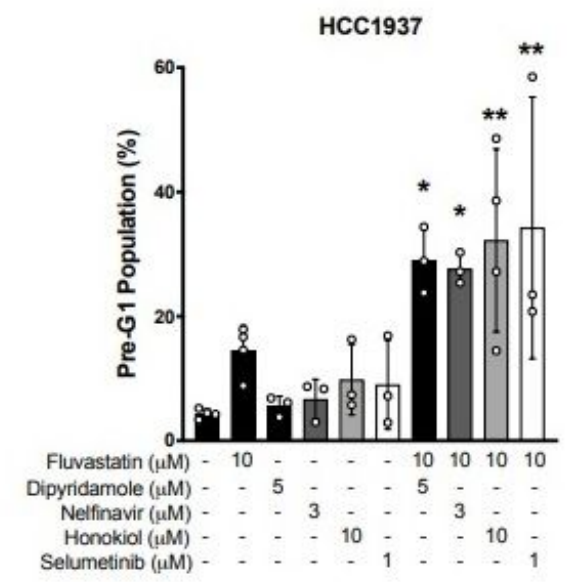

B

MDA-MB-231

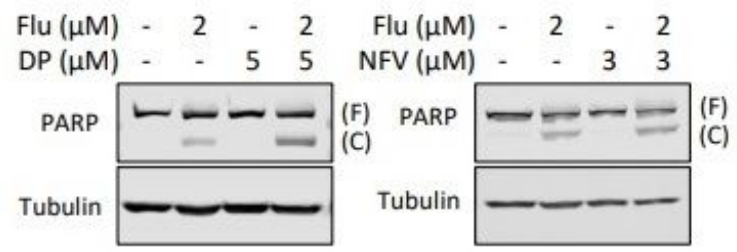

HCC1937

Flu $(\mu \mathrm{M})-10 \quad-10 \quad$ Flu $(\mu \mathrm{M})-10-10$ $\mathrm{DP}(\mu \mathrm{M}) \quad-55 \mathrm{NFV}(\mu \mathrm{M})-\quad-33$

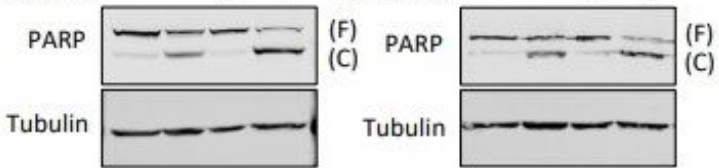

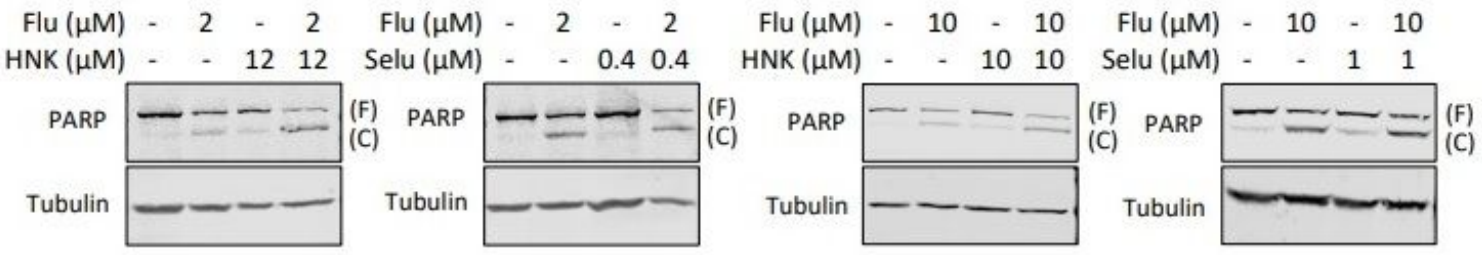

C

MDA-MB-231

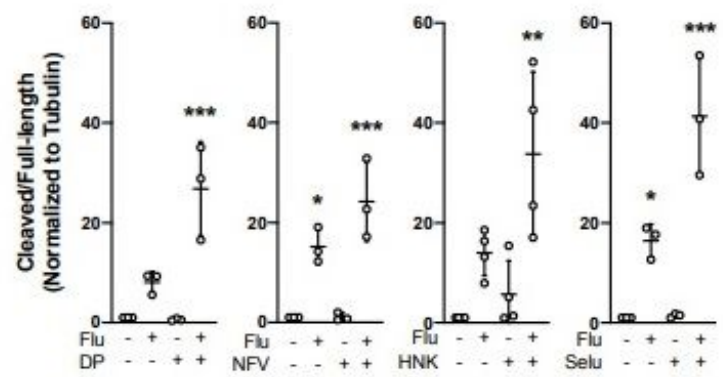

HCC1937

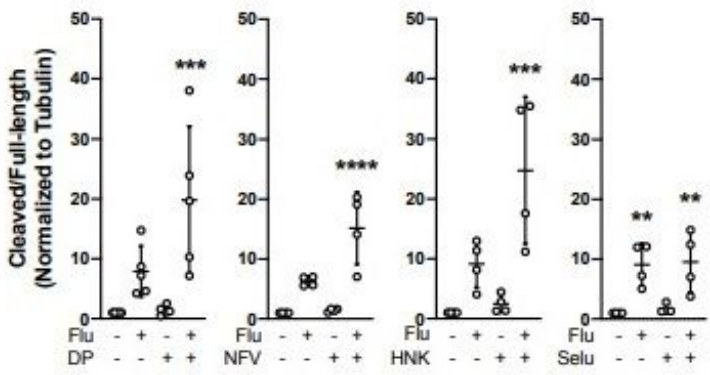

\section{Figure 2}

Dipyridamole-like compounds potentiate fluvastatin-induced cell (A) MDA-MB-231 and HCC1937 cells were treated with solvent controls or fluvastatin +/- dipyridamole (DP), nelfinavir (NFV), honokiol (HNK) or selumetinib (Selu) for 72 hours, fixed in ethanol and assayed for DNA fragmentation (\% pre-G1 population) as a marker of cell death by propidium iodide Error bars represent the mean +/- SD, $n=3-4$, * $p$ $<05,{ }^{* \star} p<0.01,{ }^{\star \star \star \star} \mathrm{p}<0.0001$ (one-way ANOVA with Bonferroni's multiple comparisons test, where each 
treatment was compared to the solvent control). (B) Cells were treated as in (A), protein isolated and immunoblotting was performed to assay for PARP (F) represents full-length PARP and (C) represents cleaved PARP. (C) PARP cleavage (cleaved/full-length) shown in (B) was quantified by densitometry and normalized to Tubulin Error bars represent the mean $+/-S D, n=3-5,{ }^{*} p<0.05,{ }^{* \star} p<0.005,{ }^{* \star *} p<0.001$, $\star \star \star \star x p<0.0001$ (one-way ANOVA with Bonferroni's multiple comparisons test, where each group was compared to the solvent control within each experiment).
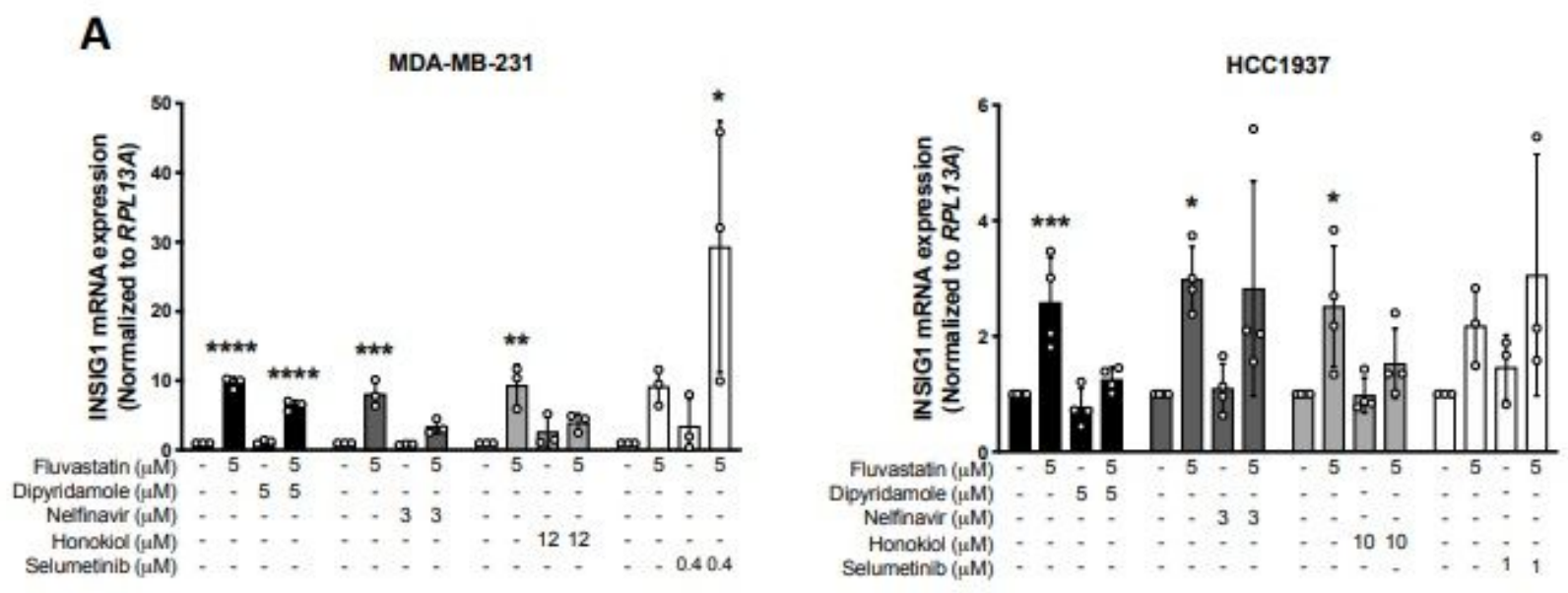

B

MDA-MB-231

HCC1937

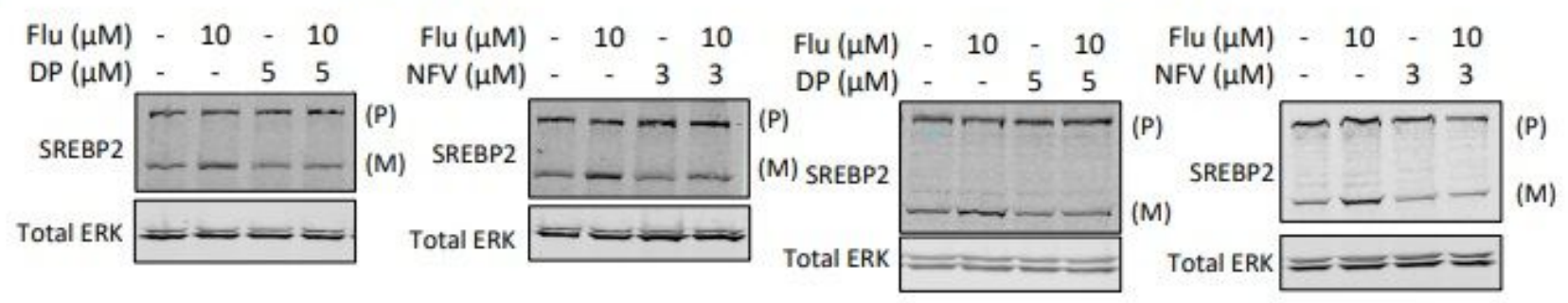

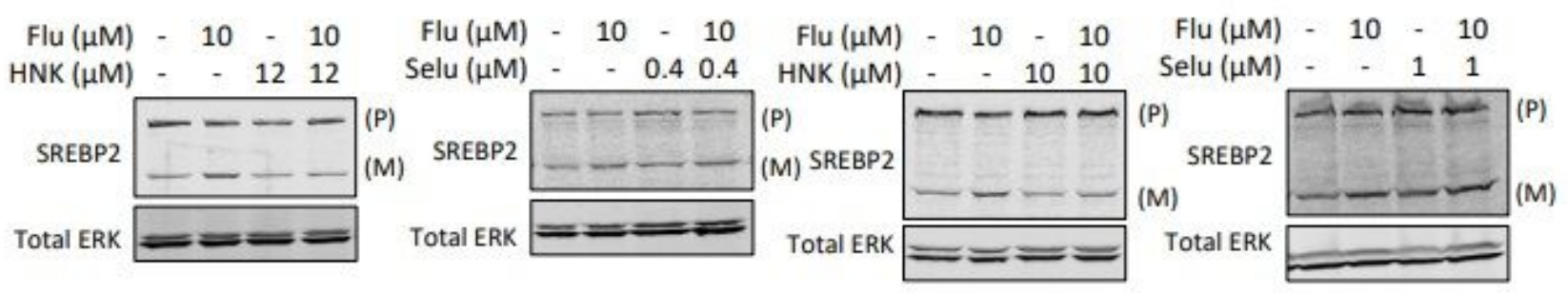

C

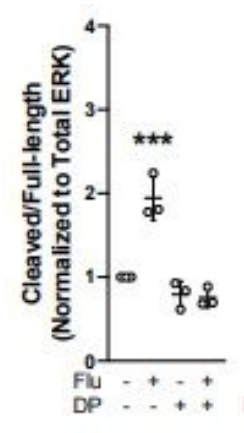

MDA-MB-231

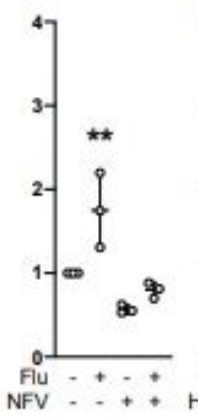

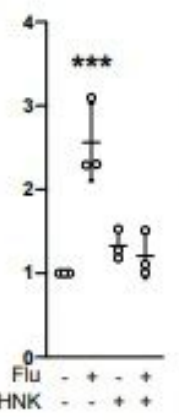
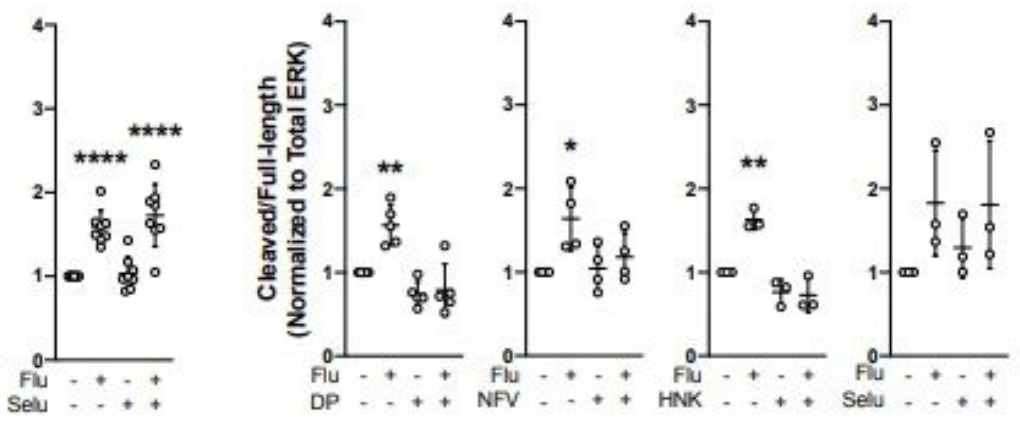

HCC1937

\section{Figure 3}


Nelfinavir and Honokiol block fluvastatin-induced SREBP (A) MDA-MB-231 and HCC1937 cells were exposed to solvent controls, fluvastatin (Flu) +/- dipyridamole (DP), nelfinavir (NFV), honokiol (HNK) or selumetinib (Selu) for 16 hours, and RNA was isolated to assay INSIG1 expression by qRT-PCR. mRNA expression data are normalized to RPL13A Error bars represent the mean +/-SD, $n=3-4$, * $<<0.05$, ${ }^{* \star} p<0.005,{ }^{* \star *} p<0.001,{ }^{* \star *} p<0.0001$ (one-way ANOVA with Bonferroni's multiple comparisons test, where each group was compared to the solvent control group within each experiment). (B) MDA-MB-231 and HCC1937 cells were treated with fluvastatin +/- dipyridamole, nelfinavir, honokiol or selumetinib for 12 hours, and protein was harvested to assay for SREBP2 expression and cleavage (activation) by (P) represents precursor, full-length SREBP2 and (M) represents mature, cleaved (C) SREBP2 cleavage (cleaved/full-length) was quantified by densitometry and normalized to total ERK Error bars represent the mean $+/-$ SD, $n=3-8,{ }^{\star} p<05, * \star p<0.005,{ }^{\star * \star} p<0.001, \star \star \star \star x p<0.0001$ (one-way ANOVA with Bonferroni's multiple comparisons test, where each group was compared to the solvent controls group within its experiment). 
A

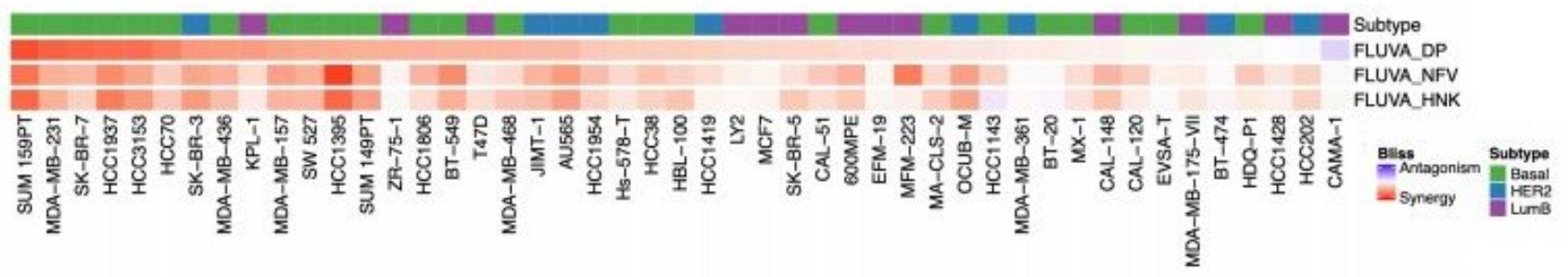

B

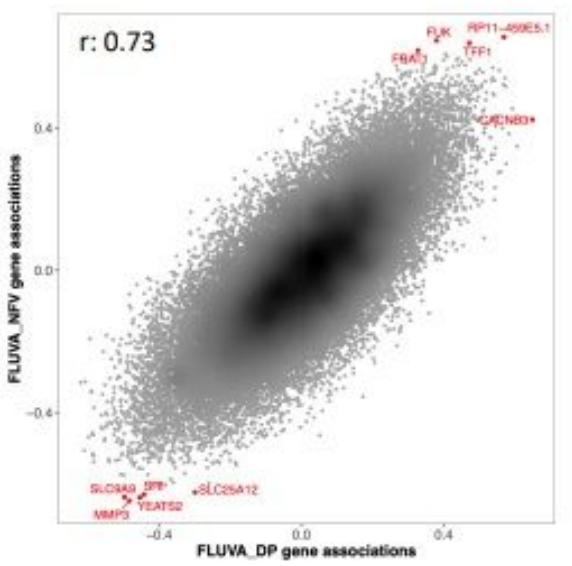

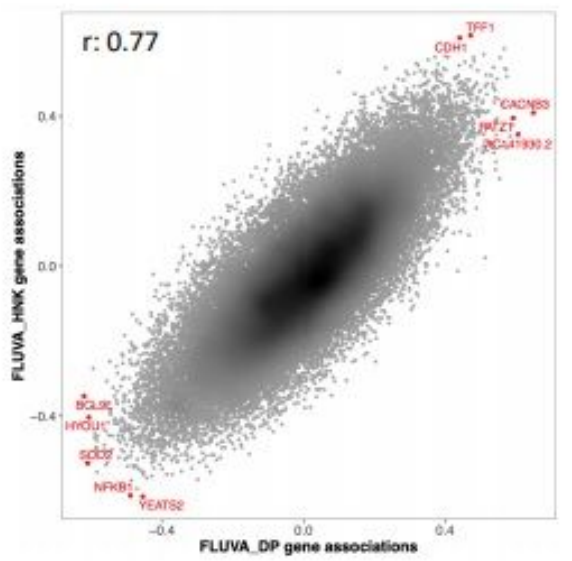

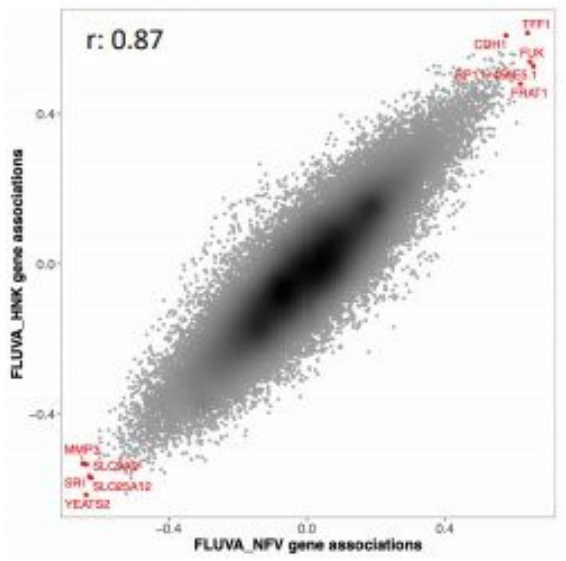

C

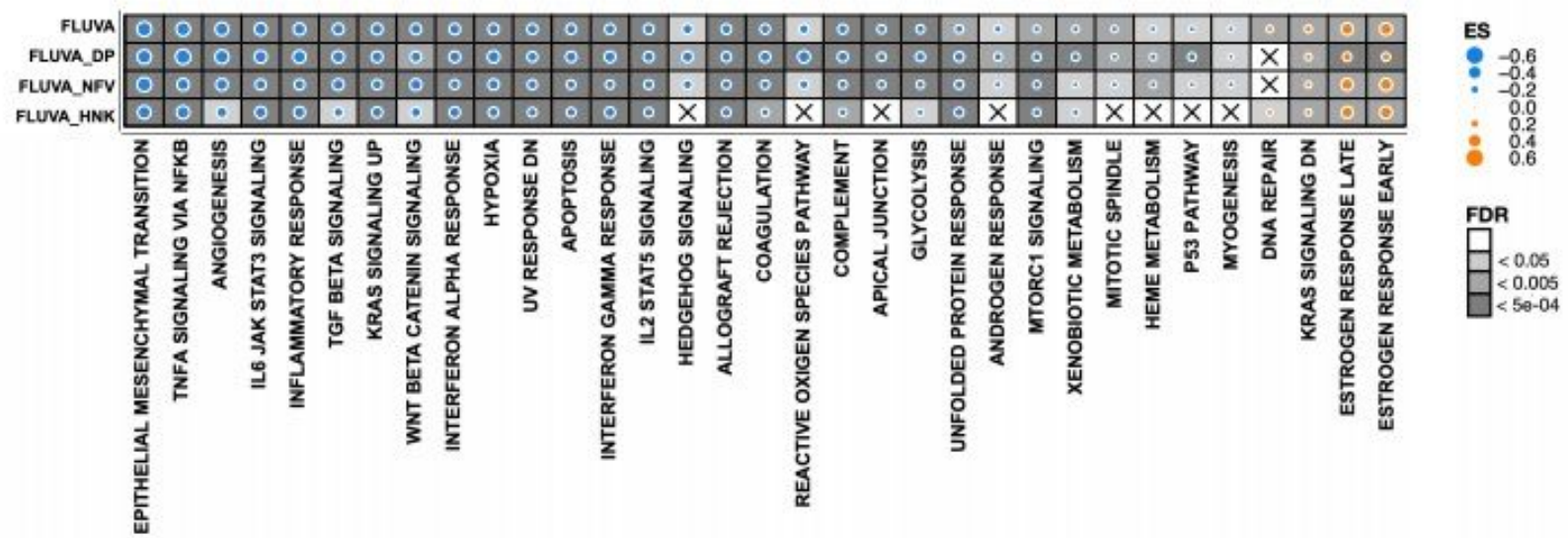

\section{Figure 4}

Compound combination synergy (A) Heatmap of synergy scores (Bliss Index model) for fluvastatin (Fluva) + dipyridamole (DP), nelfinavir (NFV) or honokiol (HNK) in a panel of 47 breast cancer cells Ordered by synergy score of Fluva-DP, from greatest $(<0)$ to least synergy $(>0)$. Breast cancer subtype of each cell line is shown and is based on the SCMOD2 subtyping (B) Basal mRNA expression34 associations with synergy scores for each drug combination (e.g. Fluva-NFV Fluva-DP, Fluva-HNK FluvaDP, and Fluva-NFV vs. Fluva-HNK). Associations were calculated using Pearson correlation Top five basally-expressed genes associated with synergy in either direction are annotated in (C) Gene set enrichment analysis (GSEA) using the Hallmark gene set collection, where genes were ranked according to their correlation to the fluvastatin IC50 (Fluva) value or to the synergy score (Fluva-DP, Fluva-NFV and Fluva-HNK). Dot plot was restricted to pathways enriched in two out of the four groups. Dot size indicates 
the difference in enrichment scores (ES) of the pathways. Background shading indicates the X indicates pathway and drug combinations that were not significantly enriched (FDR $>05)$.

A

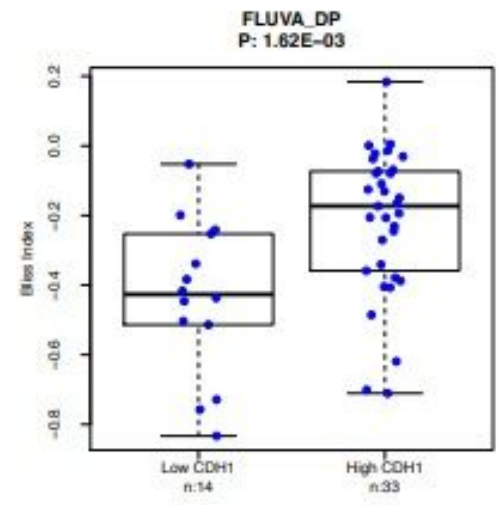

B

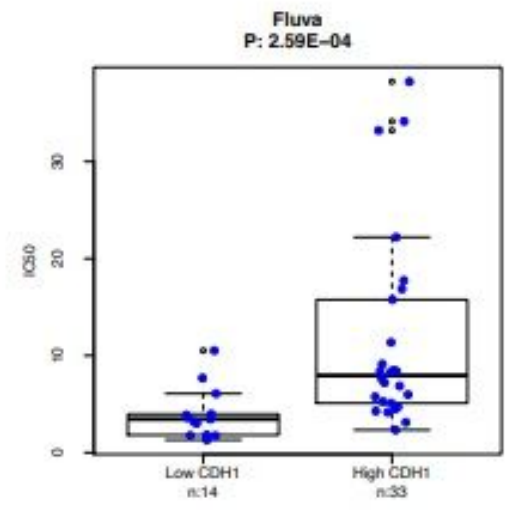

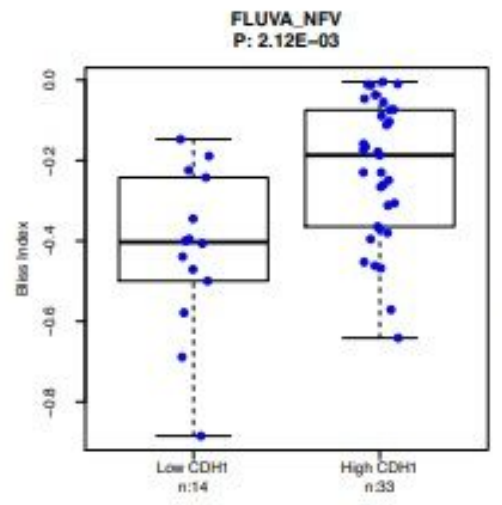

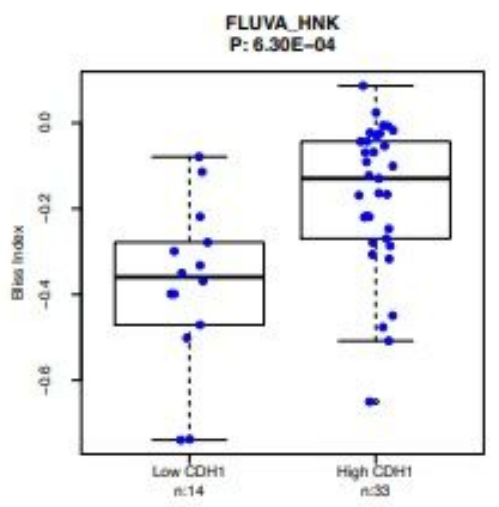

C

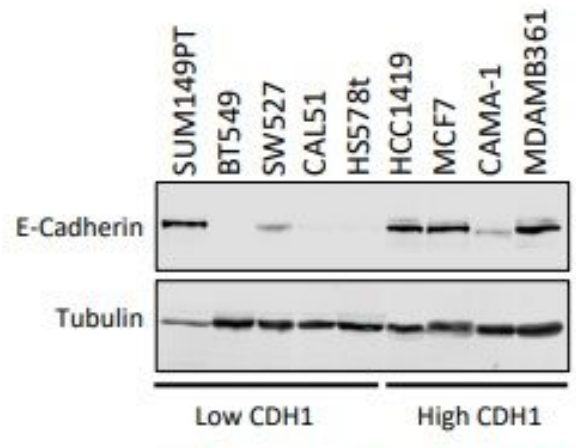

D
Protein

expression

(o tubulin)

E

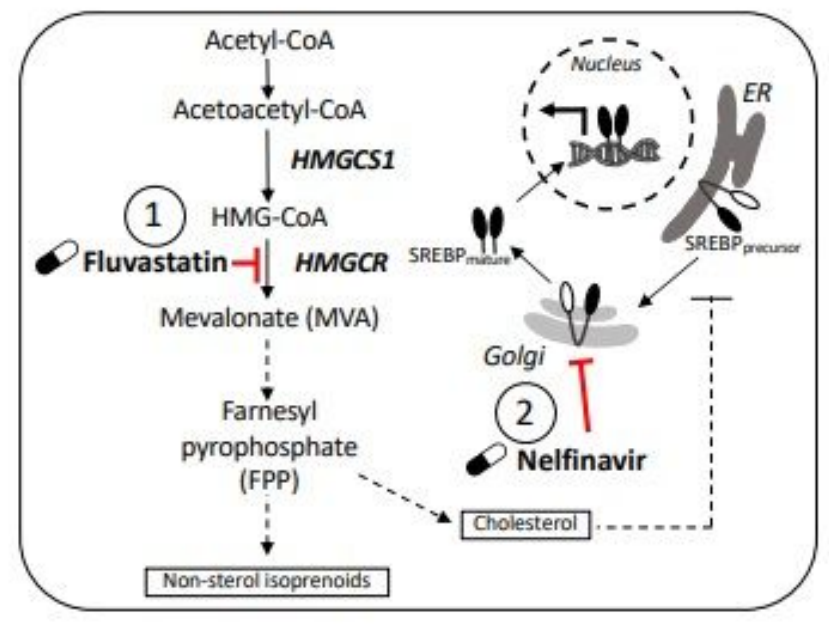

Figure 5

Basal E-cadherin predicts synergistic response to fluvastatin-compound combinations. (A) Basal Ecadherin mRNA expression between cell lines predicted to be synergistic or not to each drug Synergy was defined by Bliss Index and significance was measured by wilcoxon rank sum (B) Basal E-cadherin mRNA 
expression between cell lines predicted to be respondent or not to sensitivity was defined by IC50 and significance was measured by wilcoxon rank sum test. (C)Protein lysates were isolated from a panel of breast cancer cell lines to assay for basal E-cadherin expression by (D) Densitometry values of normalized E-cadherin expression plotted as a E-cadherin expression was quantified by densitometry and normalized individually to Tubulin (E) Schematic diagram detailing the potential for fluvastatin (labelled with 1) and nelfinavir (labelled with 2) to block the SREBP2-mediated feedback response and synergize to potentiate fluvastatin-induced cell 